\title{
CONJUGACY OF CARTER SUBGROUPS IN GROUPS OF FINITE MORLEY RANK
}

\author{
Olivier Frécon (Université De Poitiers) \\ olivier.frecon@math.univ-poitiers.fr
}

\begin{abstract}
The Cherlin-Zil'ber Conjecture states that all simple groups of finite Morley rank are algebraic. We prove that any minimal counterexample to this conjecture has a unique conjugacy class of Carter subgroups, which are defined as being the definable connected nilpotent subgroups of finite index in their normalizers, and which are analogous to Cartan subgroups in algebraic groups.
\end{abstract}

\section{INTRODUCTION}

This paper is a contribution to the study of the Cherlin-Zil'ber Conjecture, which states that the infinite simple groups of finite Morley rank are algebraic. In the view of this conjecture, the search for analogues in groups of finite Morley rank of classical theorems in affine algebraic groups has been a continuing subject. In this article, we consider the conjugacy of Cartan subgroups, which are defined in affine algebraic groups as being the connected components of the centralizers of maximal tori. These subgroups are the maximal tori in simple algebraic groups. In groups of finite Morley rank, Cartan subgroups are approximated by Carter subgroups, which are defined as being definable, connected, nilpotent, and of finite index in their normalizers. These subgroups exist in any group of finite Morley rank.

Fact 1.1. - [14] Any group of finite Morley rank has a Carter subgroup.

Carter subgroups have turned out to be increasingly useful in the analysis of groups of finite Morley rank. In particular since, contrary to other categories of subgroups, they are nontrivial as soon as the ambient group is infinite. Furthermore, the most difficult problem concerning the Cherlin-Zil'ber Conjecture is the search for an analogue with the Feit-Thompson Theorem, and few tools allow to advance in this direction. In this context, probably Carter subgroups have a role to play.

This article concerns the conjugacy of Carter subgroups in $K^{*}$-groups, in other words in the groups of finite Morley rank all of whose proper, infinite, simple, definable and connected sections are algebraic over an algebraically closed field. Indeed, for the Cherlin-Zil'ber Conjecture, it is enough to analyze just simple $K^{*}$ groups, which are the minimal potential counterexamples.

Theorem 1.2. - Carter subgroups are conjugate in each $K^{*}$-group.

\footnotetext{
Date: December 12, 2007.

${ }^{0}$ Mathematics Subject Classification 2000: 03C45, 03C60, 20A15, 20E32

${ }^{0}$ Keywords: Cherlin-Zil'ber Conjecture, groups of finite Morley rank, Carter subgroups, Cartan subgroups.
} 
Actually, this theorem is the outcome of several studies concerning the conjugacy of Carter subgroups in groups of finite Morley rank. Indeed, the conjugacy of Carter subgroups has been proven in several important particular cases (see [10, 12, 15, 19]). Notably two of these ones will be very useful for us. First F. Wagner proved the following result, which will be used all the long of this paper.

Fact 1.3. - [19] Let $G$ be a solvable group of finite Morley rank. Then any two Carter subgroups of $G$ are conjugate.

Secondly E. Jaligot proved the conjugacy of generous Carter subgroups (Definition 2.10), which is certainly the more general conjugacy result of the subject. This one will be very useful to us. Thanks to this, we have just to consider non generous Carter subgroups, which is an important restriction (see Section 4).

Fact 1.4. - [15] In any group G of finite Morley rank, generous Carter subgroups are conjugate and generically disjoint.

At the beginning, our first aim was to consider right minimal connected simple groups, which is the most important case, but it turned out that the proof of Theorem 1.2 and some remarks about [6] (Section 6) are enough to obtain the complete result, and even more. Indeed the main part of this paper concerns the proof of Theorem 1.5, then we prove Corollaries 1.6 and 1.9 (Section 5). Finally we obtain Theorem 1.2 and Corollary 1.8 as particular cases of Corollary 1.6.

We use the concept of P-groups, which are the groups of finite Morley rank all of whose definable connected simple sections have a nontrivial pseudo-torus (Definitions 4.1 and 2.17). In particular, any solvable group of finite Morley rank is a $P$-group.

Theorem 1.5. - Let $G$ be a group of finite Morley rank. If the normalizer of each nontrivial abelian divisible definable subgroup is a P-group, then the divisible Carter subgroups of $G$ are conjugate.

Moreover, if $G$ has a non generous divisible Carter subgroup $C$, then there is an interpretable field $K$ of characteristic zero such that $C$ is a homogeneous $U_{K^{-}}$ subgroup (Definition 3.8), and $G$ has no generous Carter subgroup.

Our first corollary is our most general result about the conjugacy of all the Carter subgroups in a group of finite Morley rank. Moreover it speak about the structure of a group of finite Morley rank with a non generous Carter subgroup. Its proof uses Fact 5.3.

Corollary 1.6. - Let $G$ be a group of finite Morley rank. Suppose that the normalizer of each nontrivial abelian definable subgroup is a P-group. Then its Carter subgroups are conjugate.

Moreover, if $G$ has a non generous Carter subgroup $C$, then $G^{\circ}$ is a $\widetilde{V}$-group (Definition 5.1), and $C$ is a homogeneous $U_{K}$-subgroup for an interpretable field $K$ of characteristic zero.

Now we obtain Corollary 1.8 by the following fact.

Fact 1.7. - [2, Theorem 3] Let $G$ be an infinite simple group of finite Morley rank. If any decent torus of $G$ is trivial, then $G$ contains no involutions.

Indeed, if we consider toward a contradiction such a group $G$, then Fact 1.4 provides a non generous Carter subgroup $C$, and $G$ is a $P$-group by Fact 1.7 . 
Moreover $G^{\circ}$ is a $\widetilde{V}$-group by Theorem 1.6, in particular $G$ has no nontrivial pseudotorus, so $G^{\circ}$ is solvable (Remark 4.3), contradicting Fact 2.12.

Corollary 1.8. - In any group of finite Morley rank all of whose definable simple sections have involutions, Carter subgroups are conjugate and generous.

We note that, for any $K^{*}$-group $G$, either $G$ is a minimal connected simple group, or its definable simple sections have involutions. Consequently Corollaries 1.6 and 1.8 imply Theorem 1.2.

The following result, proved in Section 5, is another justification to provide a general result as Corollary 1.6, rather than just Theorem 1.2.

Corollary 1.9. - Let $G$ be a group of finite Morley rank. Suppose that $G$ has a normal minimal connected simple subgroup $A$, such that $G / A$ is solvable. Then the Carter subgroups of $G$ are conjugate.

Our paper is organized as follows. In Section 2, some well-known results are mentionned. In Section 3, we discuss the concepts of unipotence introduced by J. Burdges into [4]. All our information on the structure of studied groups will be based on these concepts.

In Section 4, we reduce our analysis to $C^{*}$-groups, that is a minimal configuration. This section is our first very important step. However it is not very hard since the most of this restriction work has been make elsewhere. Indeed E. Jaligot proved that generous Carter subgroups are conjugate in any group of finite Morley rank (Fact 1.4), consequently our study will only concern non generous Carter subgroups. Moreover the analysis by G. Cherlin in [7] about decent tori (Definition 2.17) allows us to content our study to Carter subgroups without a nontrivial decent torus, and even without a nontrivial pseudo-torus by the generalisation of this work in [12].

In Section 5, we show that Corollaries 1.6 and 1.9 are well consequences from Theorem 1.5. Henceforth, we have just to prove Theorem 1.5 for $C^{*}$-groups.

In Section 6, we yield the results of [6] needed here. This work by J. Burdges is essential for us. Indeed, several times it will allow to destroy very bad configurations, for example in the proof of Theorem 7.3. Actually, the facts of this section are written in [6] for minimal connected simple groups. However, their proofs in [6] allows the more general versions given here.

In Section 7, we consider the structure of a non generous divisible Carter subgroup. The main result of this section is nontrivial and highly necessarily for all the rest of this paper, and it starts our general strategy for the complete proof of Theorem 1.5. Indeed, our main idea is, if the structure of Carter subgroup resemble to the one of an algebraic torus, for example it is a pseudo-torus (Definition 2.17), then it is generous (see Fact 2.21). Hence a non generous Carter subgroup $C$ has to be "unipotent-like". This is essential since our final rank calculation will be feasible just if we obtain a sufficiently clear, and homogeneous, configuration. Henceforth our strategy is the following:

- we show that the structure of a non generous divisible Carter subgroup is "unipotent" in a strong sense including the homogeneity (Theorem 7.3);

- we show that any subgroup with a nontrivial intersection with such a Carter subgroup has this unipotent-like structure too (Proposition 8.2);

- we study the intersections between Carter subgroups in the terms of subgroups $V_{K}(\cdot)$ (Sections 9 and 10). Note that the intersections of Carter subgroups will be characterized, in a certain sense, as the subgroups $H$ such that $H=V_{K}(H)$ 
(Corollary 9.6), and their analysis culmins with Theorem 10.5, which shows that, under some conditions, these subgroups are generically "disjointed". This work is again very necessarily for the final argument.

- from now on, this structural analysis enables us to make the finale rank calculation, and to obtain a genericity theorem (Theorem 10.6), then Theorem 1.5 (Section 11). At this stage, all the arguments are relatively formal.

\section{FACTS}

The notations will be as in [3], which is also our basic reference. In this section we recall definitions and known results.

2.1. General points. If $X$ is a subset of a group $G$ of finite Morley rank, then the definable closure of $X$, denoted by $d(X)$, is the intersection of all the definable subgroups of $G$ which contain $X$. By the descending chain condition on definable subgroups, this intersection is definable.

If $H$ is a subgroup of a group $G$ of finite Morley rank, the connected component of $H$, denoted by $H^{\circ}$, is $H \cap d(H)^{\circ}$.

Fact 2.1. - [3, Proposition 6.18 and Theorem 6.19] Let $P$ be a locally finite $p$ subgroup of a group $G$ of finite Morley rank, where $p$ is a prime. Then $P^{\circ}=B * T$ is the central product of a nilpotent subgroup $B$ of bounded exponent and a p-torus T. In particular $P^{\circ}$ is nilpotent.

Fact 2.2. - [3, Theorem 9.29] Let $G$ be a connected solvable group of finite Morley rank. Then the Sylow p-subgroups of $G$ are connected for any prime $p$.

Fact 2.3. - [3, Corollary 6.12] Let $G$ be a nilpotent group of finite Morley rank. Then $G$ is a central product $G=D * C$ where $D \cap C$ is finite, and

- $D$ is definable, connected, characteristic and divisible;

- $C$ is definable, characteristic and of bounded exponent;

- the torsion part of $D$ is divisible and central, and $D^{\prime}$ is torsion-free.

The Fitting subgroup $F(G)$ of a group $G$ is the subgroup generated by all the normal nilpotent subgroups of $G$. Nesin proved that the Fitting subgroup is definable and nilpotent in any group of finite Morley rank [3, Theorem 7.3].

Fact 2.4. - [3, Theorem 9.21] Let $G$ be a connected and solvable group of finite morley rank. Then $G / F(G)^{\circ}$ (so also $G / F(G)$ ) is a divisible abelian group.

A subgroup $A$ of a group $G$ of finite Morley rank is said to be $S$-minimal, where $S$ is a subset of $G$, if $A$ is infinite, definable, normalized by $S$ and minimal for these conditions.

Fact 2.5. - [3, Proposition 7.7] Let $A$ be a G-minimal subgroup of a connected group $G$ of finite Morley rank. Then either $A$ is abelian, or $Z(A)$ is finite and $A / Z(A)$ is simple.

2.2. Fields of finite Morley rank. The following result due to B. Zil'ber confers on fields of finite Morley rank a central importance. Note that, by a theorem of A. Macintyre [3, Theorem 8.1], an infinite field of finite Morley rank is always algebraically closed. 
Fact 2.6. - [3, Theorem 9.1] Let $G=A \rtimes H$ be a group of finite Morley rank where $A$ and $H$ are two infinite definable abelian subgroups, $A$ is $H$-minimal and $C_{H}(A)=1$. Then $G$ interprets an algebraically closed field $K$ such that $A \cong K_{+}$ definably, $H$ is definably isomorphic to a subgroup of $K^{\times}$.

Fact 2.7. - [17, Corollaire 3.3] Let $K$ be a field of finite Morley rank of characteristic 0 . Then $K_{+}$has no nontrivial proper definable subgroup.

The following fact due to F. Wagner will be very used to eliminate the torsion in our restriction to $C^{*}$-groups (Section 4$)$.

Fact 2.8. - [18] Let $K$ be a field of finite Morley rank of positive characteristic $p$. Then each definable subgroup of $K^{\times}$is the definable closure of its torsion.

Fact 2.9. - [11, Proposition 5.7] For any divisible nilpotent group G of finite Morley rank, the following conditions are equivalent:

- There is a uniformly definable family $\mathscr{F}$ of proper sugroups of $G$ such that $\cup \mathscr{F}$ is a generic subset of $G$;

- There is an interpretable algebraically closed field $K$ and a normal definable subgroup $M$ of $G$ such that $G / M$ is definably isomorphic to a $K$-vector space of dimension 2.

2.3. Generosity, Carter subgroups and generalized centralizers. In [15], E. Jaligot introduced the following notion, which is very important for the analysis of Carter subgroups. Indeed, for the conjugacy problem, by the main result of [15] (Fact 1.4), we have just to study non generous Carter subgroups.

Definition 2.10. - A definable subset $X$ of a group $G$ of finite Morley rank is generous if the union of its conjugates is a generic subset of $G$.

Fact 2.11. - [15, Lemma 3.9] Let $G$ be a group of finite Morley rank with two definable subgroups $H$ and $L$. If $L$ is a generous subgroup of $H$ and if $H$ is a connected generous subgroup of $G$, then $L$ is a generous subgroup of $G$.

In solvable groups, the behavior of Carter subgroups is well understood, notably by Fact 1.3 and by the following results.

Fact 2.12. - [8, Lemma 3.5] Let $G$ be a solvable group of finite Morley rank. Then the Carter subgroups of $G$ are generically disjoint, in particular any Carter subgroup of $G$ is generous.

Fact 2.13. - [13, Corollaire 5.20] Let $G$ be a connected solvable group of finite Morley rank and $N$ a normal definable subgroup of $G$. Then Carter subgroups of $G / N$ are exactly of the form $Q N / N$, with $Q$ a Carter subgroup of $G$.

If $G$ is a group and $X$ a subset of $G$, the generalized centralizer $E_{G}(X)$ of $X$ in $G$ is the set of elements $g \in G$ such that, for each $x \in X,\left[g,_{n} x\right]=1$ for some $n \in \mathbb{N}$, where $[g, 0 x]=g$ and $\left[g,_{n+1} x\right]=\left[\left[g,_{n} x\right], x\right]$.

Fact 2.14. - [13, Corollaire 5.17] Let $G$ be a connected solvable group of finite Morley rank and $H$ a nilpotent subgroup of $G$. Then $E_{G}(H)$ is a definable connected subgroup of $G$ and $H \leq F\left(E_{G}(H)\right)$.

Fact 2.15. - [13, Théorème 1.1 and Section 7.2] Let $G$ be a connected solvable group of finite Morley rank and $H$ a nilpotent subgroup of $G$. 
- $E_{G / N}(H N / N)=E_{G}(H) N / N$ for every normal subgroup $N$ of $G$.

- $E_{G}(H)$ contains a Carter subgroup of $G$.

Fact 2.16. - [13, Théorème 7.9] Let $G$ be a connected solvable group of finite Morley rank. Then there are at most $2^{r k(G)}$ conjugacy classes of generalized centralizers of nilpotent subgroups.

2.4. Decent tori and pseudo-tori. In [7], G. Cherlin defines decent tori as an analogue to tori algebraic. In [12], we introduce pseudo-tori, as a more general notion, independant of the torsion. Here we relate just the more general results used in this paper, and concerning pseudo-tori, but their proof are very often similar to their analogue in [7] concerning decent tori.

Note that the concept of decent torus is sufficient to prove Theorem 1.2, since we did not use pseudo-tori in the first version of this paper. In fact, we prefer use pseudo-tori rather than decent tori just because we obtain more precise structural information about a possibly non generous Carter subgroup.

Definition 2.17. - Let $T$ be a divisible abelian group of finite Morley rank. We say that $T$ is a decent torus if $T$ is the definable closure of its torsion.

We say that $T$ is a pseudo-torus if no definable quotient of $T$ is definably isomorphic to $K_{+}$for an interpretable field $K$.

Fact 2.18. - [12, Proposition 2.7] Let $G$ be a connected group of finite Morley rank acting by conjugation on a pseudo-torus $T$. Then $G$ centralizes $T$.

Fact 2.19. - [12, Corollary 2.8] Let $G$ be a nilpotent group of finite Morley rank. Then $G$ has a unique maximal pseudo-torus.

Fact 2.20. - [12, Corollary 2.10] Let $G$ be a group of finite Morley rank. Then any pseudo-torus of $G$ is contained in a Carter subgroup of $G$.

Fact 2.21. - [12, Corollary 2.12] Let $T$ be a pseudo-torus of a connected group $G$ of finite Morley rank. Then $C_{G}(T)$ is connected and generous in $G$.

Fact 2.22. - [12, Corollary 2.13] Let $G$ be a group of finite Morley rank, $N$ a normal definable subgroup of $G$ and $T$ a maximal pseudo-torus of $G$. Then $T N / N$ is a maximal pseudo-torus of $G / N$ and every maximal pseudo-torus of $G / N$ has this form.

\section{UNipotence}

In [4], J. Burdges introduced some analogues of algebraic unipotence for groups of finite Morley rank. In [9], we continued the analysis of these concepts. Here are just given the more general results used in the article, and we refer to [4], or [9], for a more complete introduction concerning these unipotence notions.

3.1. $U_{0, r}$-groups. For a notion analogous to unipotence in algebraic groups, Burdges [4] introduced the notions of reduced rank and $U_{0, r}$-groups.

An abelian connected group $A$ of finite Morley rank is indecomposable if it is not the sum of two proper definable subgroups. If $A \neq 1$, then $A$ has a unique maximal proper definable connected subgroup $J(A)$, and if $A=1$, let $J(1)=1$.

Fact 3.1. - [12, Lemma 2.2] Let $G$ be a group of finite Morley rank and $H$ a definable normal subgroup of $G$. If $\bar{B}$ is a divisible indecomposable subgroup of $G / H$, then there is an indecomposable subgroup $A$ of $G$ such that $\bar{B}=A H / H$. 
In particular, Fact 3.1 says that any divisible group of finite Morley rank is generated by its indecomposable subgroups.

As in [4], the reduced rank of any abelian group $A$ of finite Morley rank is $\bar{r}(A)=r k(A / J(A))$. Also, if $G$ is any group of finite Morley rank and $r$ a positive integer, then we define

$U_{0, r}(G)=\langle A \leq G| A$ is indecomposable, $\bar{r}(A)=r, A / J(A)$ is torsion-free $\rangle$

We say that $G$ is a $U_{0, r}$-group whenever $G=U_{0, r}(G)$.

Remark 3.2. - By Fact 2.3, any indecomposable subgroup is either of bounded exponent, either a $U_{0, r}$-group for $r$ a positive integer, or a decent torus.

For every nonzero integer $r$, a Sylow $U_{0, r}$-subgroup of a group $G$ of finite Morley rank is a maximal nilpotent $U_{0, r}$-subgroup of $G$.

Fact 3.3. - [5, Lemma 6.2] Let $G$ be a group of finite Morley rank. Then, for each nonzero integer $r$, the Sylow $U_{0, r}$-subgroups of $G$ are exactly those nilpotent $U_{0, r}$-subgroups $S$ such that $U_{0, r}\left(N_{G}(S)\right)=S$.

Fact 3.4. - [5, Theorem 6.5] Let $G$ be a solvable group of finite Morley rank. Then the Sylow $U_{0, r}$-subgroups of $G$ are conjugate for each nonzero integer $r$.

Fact 3.5 is not written in [14], but it is deduced of the proof of Fact 1.1.

Fact 3.5. - [14] Let $G$ be a group of finite Morley rank. If $r$ is an integer and if $S$ is a Sylow $U_{0, r}$-subgroup of $G$ such that $N_{G}(S)$ has no nontrivial decent torus and such that $U_{0, s}\left(N_{G}(S)\right)=1$ for each $s<r$, then $S$ is contained in a Carter subgroup of $G$ as a normal subgroup.

Fact 3.6. - [14] Let $G=N C$ be a group of finite Morley rank where $N$ and $C$ are nilpotent definable connected subgroups and $N$ is normal in $G$. Assume that there is an integer $n \geq 1$ such that $N=\left\langle U_{0, s}(N) \mid 1 \leq s \leq n\right\rangle$ and $C=\left\langle U_{0, s}(C) \mid s \geq n\right\rangle$. Then $G$ is nilpotent.

3.2. $\widetilde{U}$-groups. We introduce the notion of $\widetilde{U}$-groups as a more precise unipotence notion than $U$-groups defined in [9]. This concept depend on pseudo-tori [12].

Notation 3.7. - For any interpretable field $K$ of characteristic zero and any group $G$ of finite Morley rank, we denote by $U_{K}(G)$ the (definable connected) subgroup of $G$ generated by its indecomposable subgroups $A$ such that $A / J(A)$ is definably isomorphic to $K_{+}$.

Definition 3.8. - A group $G$ of finite Morley rank is said to be a $U_{K}$-group, where $K$ is an interpretable field of characteristic zero, if $G=U_{K}(G)$. We say that a $U_{K}$-group $G$ is homogeneous if each definable connected subgroup of $G$ is a $U_{K}$-subgroup.

Moreover, for every group $G$ of finite Morley rank, we denote by $\widetilde{U}(G)$ the subgroup of $G$ generated by its normal homogeneous $U_{K}$-subgroups, for the interpretable fields $K$ of characteristic zero, and by its normal definable connected subgroups of bounded exponent.

A $\widetilde{U}$-group is a group $G$ of finite Morley rank such that $G=\widetilde{U}(G)$.

Remark 3.9. - By Fact 2.7, any divisible indecomposable subgroup is either a pseudo-torus or a $U_{K}$-group for an interpretable field $K$ of characteristic 0 .

By Fact 2.22 , in any $\widetilde{U}$-group, each pseudo-torus is trivial. 
We do not write the proofs of the following results. Indeed, by using [12] instead of [7], they are similar to the ones of $[4,5,9]$.

Fact 3.10. - (Compare with [5, Theorem 3.4]) Let $G$ be a divisible nilpotent group of finite Morley rank, and let $T$ be its maximal pseudo-torus. Then $G$ interprets some fields $K_{1}, \cdots, K_{n}$ of characteristic zero such that

$$
G=T * U_{K_{1}}(G) * U_{K_{2}}(G) * \cdots * U_{K_{n}}(G)
$$

Fact 3.11. - (Compare with [9, Theorem 4.11]) Let $G$ be a connected group of finite Morley rank, and $K$ an interpretable field of characteristic 0. Assume that $G$ acts definably by conjugation on $H$, a nilpotent $U_{K}$-group. Then $[G, H]$ is a homogeneous $U_{K}$-subgroup.

Fact 3.12. - (Particular case of $[9$, Proposition 5.3]) Any solvable $\widetilde{U}$-group is nilpotent.

Fact 3.13. - (Compare with $[9$, Theorem 5.4]) Let $G$ be a nilpotent $\widetilde{U}$-group. Then $G$ interprets some algebraically closed fields $K_{1}, \cdots, K_{n}$ of characteristic zero such that the following decomposition holds:

$$
G=B \times U_{K_{1}}(G) \times U_{K_{2}}(G) \times \cdots \times U_{K_{n}}(G)
$$

where $B$ is a definable connected characteristic subgroup of bounded exponent, and $U_{K_{s}}(G)$ a homogeneous $U_{K_{s}}$-subgroup (for $s \in\{1,2, \ldots, n\}$ ).

Fact 3.14. - (Compare with $[9$, Corollary 5.6]) Let $G$ be a nilpotent group of finite Morley rank. Then $\widetilde{U}(G)$ is the largest $\widetilde{U}$-subgroup of $G$.

Fact 3.15. - (Compare with [9, Proposition 5.7]) Let $G$ be a torsion-free group of finite Morley rank without a nontrivial pseudo-torus. Then $G$ is a $\widetilde{U}$-group if and only if, for each interpretable field $K$ of characteristic $0, U_{K}(G)$ is a homogeneous $U_{K}$-subgroup.

Fact 3.16. - (Compare with [9, Corollary 5.8])

- Every definable quotient of a $\widetilde{U}$-group is a $\widetilde{U}$-group.

- Every definable connected subgroup of a $\widetilde{U}$-group is a $\widetilde{U}$-group.

Corollary 3.17. - Let $G$ be a connected nilpotent group of finite Morley rank. Then $G$ is a $\widetilde{U}$-group if and only if its indecomposable subgroups are $\widetilde{U}$-groups.

Proof - By Fact 3.16, we may assume that each indecomposable subgroup of $G$ is a $\widetilde{U}$-group. Then $\widetilde{U}(G)$ contains each indecomposable subgroup of $G$ (Fact 3.14). As $G / \widetilde{U}(G)$ is divisible (Fact 2.3), Facts 3.1 yields $G=\widetilde{U}(G)$.

Fact 3.18. - (Compare with [4, Lemma 2.11]) Let $G$ be a group of finite Morley rank, $U$ and $V$ two definable subgroups with $V$ normal in $G$, and $K$ an interpretable field of characteristic 0. Then $U_{K}(U V / V)=U_{K}(U) V / V$.

3.3. The kernel $\widetilde{K}(G)$. The notion of $\widetilde{U}$-groups induces the following kernel.

Notation 3.19. - For any group $G$ of finite Morley rank, let $\widetilde{K}(G)$ denote the intersection of its normal definable subgroups $H$ such that $G / H$ is a $\widetilde{U}$-group.

Lemma 3.20. - Let $G$ be a torsion-free nilpotent group of finite Morley rank without a nontrivial pseudo-torus. Then $G / \widetilde{K}(G)$ is a $\widetilde{U}$-group and, if $A$ is a normal definable subgroup of $G$, then $\widetilde{K}(G / A)=\widetilde{K}(G) A / A$. 
Proof - We prove that $G / \widetilde{K}(G)$ is a $\widetilde{U}$-group. By Fact 2.22 , we may assume that $G$ has two normal definable subgroups $H$ and $K$ such that $H \cap K=1$, and such that $G / H$ and $G / K$ are $\widetilde{U}$-groups. By Facts 3.15 and 3.16 , we may assume that $G$ is a $U_{K}$-group for an interpretable field $K$ of characteristic 0. By Facts 3.15 and $3.18, G / H$ and $G / K$ are homogeneous $U_{K}$-groups. Therefore, if $A$ is a definable subgroup of $G$, then $A /(A \cap H) \cong A H / H$ is a $U_{K}$-group and Fact 3.18 gives $A=U_{K}(A)(A \cap H)$. In the same way, we have $\left.A \cap H=U_{K}(A \cap H)((A \cap H) \cap K)\right)$, so $A=U_{K}(A)$ is a $U_{K}$-group and $G$ is a $\widetilde{U}$-group.

Let $A$ be a normal definable subgroup of $G$. By the preceding paragraph, $G / \widetilde{K}(G)$ is a $\widetilde{U}$-group, and Fact 3.16 says that $(G / A) /(\widetilde{K}(G) A / A)$ is a $\widetilde{U}$-group. Thus we have $\widetilde{K}(G / A) \leq \widetilde{K}(G) A / A$. But, if $K_{G} / A=\widetilde{K}(G / A)$, then $G / K_{G}$ is a $\widetilde{U}$-group too, so $\widetilde{K}(G / A)=\widetilde{K}(G) A / A$.

Corollary 3.21. - Let $G$ be a torsion-free nilpotent group of finite Morley rank without a nontrivial pseudo-torus, and $H$ be a definable subgroup of $G$. Then $\widetilde{K}(G)$ contains $\widetilde{K}(H)$.

Proof - By Lemma 3.20, $G / \widetilde{K}(G)$ is a $\widetilde{U}$-group, therefore $H \widetilde{K}(G) / \widetilde{K}(G)$ is a $\widetilde{U}$-group too (Fact 3.16 ). Hence $\widetilde{K}(H)$ is contained in $\widetilde{K}(G)$.

Fact 3.22. - (Compare with [9, Results 5.8, 6.12 and 6.20]) Let $G$ be a solvable connected group of finite Morley rank. Then $F(G) / Z(G)$ is a $\widetilde{U}$-group.

Corollary 3.23. - Let $G$ be a solvable connected group of finite Morley rank. Then $\widetilde{K}(F(G))$ is contained in $Z(G)$

Lemma 3.24. - Let $G$ be a torsion-free solvable group of finite Morley rank without a nontrivial pseudo-torus, and $C$ a Carter subgroup of $G$. Then $\widetilde{K}(C)$ contains $\widetilde{K}(F(G))$.

ProOF - We proceed by induction on $r k(G)$. We may assume $\widetilde{K}(F(G)) \neq 1$. Let $A$ be a $G$-minimal subgroup of $\widetilde{K}(F(G))$. By Fact $2.22, G / A$ has no nontrivial pseudo-torus. By Corollary 3.21, by induction hypothesis and by Fact 2.13 , we have $\widetilde{K}(F(G) / A) \leq \widetilde{K}(F(G / A)) \leq \widetilde{K}(C A / A)$. Then Lemma 3.20 gives $\widetilde{K}(F(G)) \leq$ $\widetilde{K}(C A)=\widetilde{K}(C) A$ and $\widetilde{K}(F(G))=A(\widetilde{K}(F(G)) \cap \widetilde{K}(C))$. Thus we may assume that $\widetilde{K}(F(G)) \cap \widetilde{K}(C)$ contains no $G$-minimal subgroup. By Corollary 3.23 , this means $\widetilde{K}(F(G)) \cap \widetilde{K}(C)=1$ and $A=\widetilde{K}(F(G)) \leq Z(G) \leq C$, in particular $A$ has no nontrivial proper definable subgroup.

By Corollary 3.17 there is an indecomposable subgroup $B$ in $F(G)$, such that $B$ is not a $\widetilde{U}$-group. Therefore we have $1 \neq \widetilde{K}(B) \leq \widetilde{K}(F(G))=A$ (Lemma 3.20 and Corollary 3.21) and $A=\widetilde{K}(B) \leq B$ by the preceding paragraph. If $[G, B]=1$, then we have $B \leq Z(G) \leq C$, and Corollary 3.21 gives $A=\widetilde{K}(B) \leq \widetilde{K}(F(G)) \cap \widetilde{K}(C)=$ 1 , hence $[G, B]$ is nontrivial.

Let $H$ be a $G$-minimal subgroup in $[G, B]$. Then, by Lemma 3.20 and Corollary 3.21, we have $\widetilde{K}(F(G)) H / H=\widetilde{K}(F(G) / H) \leq \widetilde{K}(F(G / H))$. By induction hypothesis and by Fact 2.13 , we obtain $\widetilde{K}(F(G / H)) \leq \widetilde{K}(C H / H)$, and Lemma 3.20 gives $A=\widetilde{K}(F(G)) \leq \widetilde{K}(C) H$. Since $H$ is central in $F(G)$ by $G$-minimality, and since Facts 2.4 and 2.13 yields $G=F(G) C$, the subgroup $C \cap H$ is normal in $G$ and we have either $C \cap H=1$ or $H \leq C$. In the first case, we obtain $A \leq C \cap \widetilde{K}(C) H=\widetilde{K}(C)$, so we may assume $\bar{H} \leq C$. 
We may assume $A \not \leq \widetilde{K}(C)$. By the first paragraph, we have $A \cap \widetilde{K}(C)=1$ and, since $A \leq \widetilde{K}(C) H$, there is a definable isomorphism between $A$ and $\widetilde{K}(C) A \cap H \leq$ $H$. Since $G$ is torsion-free, $B$ is divisible. Then, since $G$ has no nontrivial pseudotorus and since $B$ is indecomposable, there is an interpretable field $K$ such that $B$ is a $U_{K}$-subgroup. But, by Fact 3.11, $\left[G, U_{K}(F(G)]\right.$ is a homogeneous $U_{K}$-group, so $H$ is a homogeneous $U_{K}$-group. This proves that $A$ is a homogeneous $U_{K}$-group. By Lemma 3.20 and Fact $3.16, B / A \leq F(G) / A$ is a $\widetilde{U}$-group and, by Fact 3.15 , $B / A$ is a homogeneous $U_{K}$-group. Now, by Fact 3.18 , for each definable subgroup $L$ of $B$, we have $L=U_{K}(L)(A \cap L)$ and, since $A$ is a homogeneous $U_{K}$-group, $L$ is a $U_{K}$-group. Hence $B$ is a homogeneous $U_{K}$-group, contradicting our choice of $B$.

\section{4. $C^{*}$-GROUPS}

In this section we show that it is enough to prove Theorem 1.5 for $C^{*}$-groups (Definition 4.4, Theorem 4.14). We define P-groups, $C$-groups and $C^{*}$-groups.

Definition 4.1. - A P-group is a group of finite Morley rank all of whose definable connected simple sections have a nontrivial pseudo-torus.

Example 4.2. - Any solvable group of finite Morley rank is a $P$-group.

Any $K^{*}$-group of finite Morley rank is either a minimal connected simple group, or a $P$-group (Fact 2.22).

Remark 4.3. - Each definable section of a $P$-group is a $P$-group.

If a $P$-group has no nontrivial pseudo-torus, then its connected component is solvable (Fact 2.22). In particular, by Facts 2.21 and 2.22, the subgroup $C_{G}(T)^{\circ}$ is solvable for any maximal pseudo-torus $T$ of a $P$-group $G$.

Consequently, by Facts 2.11, 2.12, 2.18, 2.19, 2.20 and 2.21, any $P$-group $G$ has a generous Carter subgroup.

Definition 4.4. - We say that a connected P-group $G$ is a $C$-group if, in each definable section $H / K$ of $G$, the Carter subgroups of $H / K$ are conjugate.

$A C^{*}$-group is a connected group $G$ of finite Morley rank such that,

- its proper connected definable P-sections are C-sections;

- $G$ has a non generous torsion-free Carter subgroup, with no nontrivial pseudo-torus;

- G has no nontrivial normal proper P-subgroup;

- for any infinite abelian divisible definable subgroup $A, N_{G}(A)$ is a P-group.

In particular, any connected solvable group is a $C$-group (Fact 1.3). Moreover, if a minimal connected simple group of finite Morley rank has two non conjugate Carter subgroups, then it is a $C^{*}$-group (Facts 1.3 and 1.4, and Proposition 4.7). Theorem 4.14 justifies the introduction of these concepts.

Remark 4.5. - Each definable connected section of a $C$-group is a $C$-group.

By Remark 4.3, Carter subgroups are generous in each definable section of a $C$-group, in particular $C^{*}$-groups are not $C$-groups.

Lemma 4.6. - Let $B$ be a maximal p-unipotent subgroup of a group $G$ of finite Morley rank. Suppose that $H=B \cap B_{0}$ is an intersection between $B$ and another maximal p-unipotent subgroup $B_{0} \neq B$ of $G$. If we have $r k\left(B_{1} \cap B\right) \leq r k(H)$ for every maximal p-unipotent subgroup $B_{1} \neq B$ of $G$, then $N_{G}(H)^{\circ}$ is not solvable. 
ProOF - If $N_{G}(H)^{\circ}$ is solvable, there is a unique maximal $p$-unipotent subgroup $B_{1}$ in $N_{G}(H)^{\circ}$ (Fact 2.4). By maximality of $r k(H), B$ is the unique maximal $p$ unipotent subgroup of $G$ containing $N_{B}(H)^{\circ}$, so $B$ contains $B_{1}$. This proves that $B$ contains $N_{B_{0}}(H)^{\circ}$, therefore $H$ is of finite index in $B_{0}$, and the connectedness of $B_{0}$ yields $B_{0}=H \leq B$, contradicting our hypothesis..

Proposition 4.7. - Let $G$ be a group of finite Morley rank. Suppose that, for each definable connected section $H / K$ where $H$ normalizes a nontrivial abelian definable subgroup of $G$, either $H / K$ is a $C$-group or $r k(H / K)=r k(G)$. Then every non generous Carter subgroup $C$ of $G$ is torsion-free, and has no nontrivial pseudotorus.

Proof - We may suppose that $G$ is connected and has a non generous Carter subgroup $C$. In particular, by Fact 2.12 , the group $G$ is not solvable.

Let $T$ be the maximal pseudo-torus of $C$. If $T \neq 1$, then $C$ is a Carter subgroup of $N_{G}(T)^{\circ}=C_{G}(T)^{\circ}$ (Fact 2.18). Let $T_{0}$ be a maximal pseudo-torus of $G$ containing $T$. Then we have $T_{0} \leq C_{G}(T)^{\circ}$ and $T_{0}$ is contained in a Carter subgroup $D$ of $C_{G}(T)^{\circ}$ (Fact 2.20). But $C / T$ and $D / T$ are some Carter subgroups of the $C$-group $C_{G}(T)^{\circ} / T$, so they are conjugate. By the maximality of $T$ in $C$, we obtain $T=T_{0}$ and, by the maximality of $T_{0}$ in $G$ and by Fact 2.22 , the group $C_{G}(T)^{\circ} / T$ does not have a nontrivial pseudo-torus. Thus $C_{G}(T)^{\circ} / T$, so $C_{G}(T)^{\circ}$, is solvable. By Fact 2.12, $C$ is generous in $C_{G}(T)^{\circ}$. By Facts 2.21 and 2.11, $C$ is generous in $G$, contradicting our hypothesis, hence $T=1$.

If $H / K$ is a connected definable section of $G$ with $C \leq H$ and $K$ an infinite solvable subgroup, then $H / K$ is a $C$-group. If $C K=H$, then $H / K$ is nilpotent and Fact 2.22 shows that $H / K$ has no nontrivial pseudo-torus. If $C K<H$, then $C K^{\circ}$ is a $C$-group and a Frattini Argument applied to $C K$ proves that $C K / K$ is a Carter subgroup of $H / K$, in particular $H / K$ has a Carter subgroup with no nontrivial pseudo-torus (Fact 2.22). By the conjugacy of Carter subgroups, $H / K$ has no nontrivial pseudo-torus (Fact 2.20), and $H / K$ is solvable:

If $H / K$ is a connected definable section of $G$ with $C \leq H$ and

$K$ an infinite solvable subgroup, then $H / K$ is solvable and has (*)

no nontrivial pseudo-torus.

In particular, by using Fact 2.12 and by applying $(*)$ with $H=G$, there is no normal infinite definable solvable subgroup in $G$.

From now on, we suppose that $C$ has torsion. By Fact 2.3, $C$ has a nontrivial $p$-unipotent subgroup for a prime $p$. Let $B$ be the maximal $p$-unipotent subgroup of $C$ (Fact 2.3). Then $N_{G}(B)^{\circ} / B$ is solvable by $(*)$, so $N_{G}(B)^{\circ}$ is solvable. Let $B_{0}$ be the maximal $p$-unipotent subgroup of $N_{G}(B)^{\circ}$ (Fact 2.4). If $B \neq B_{0}$, there is a $N_{G}(B)^{\circ}$-minimal subgroup $A_{0} / B$ in $B_{0} / B$ and $N_{G}(B)^{\circ} / C_{N_{G}(B)^{\circ}}\left(A_{0} / B\right)$ is a decent torus (Facts 2.6 and 2.8). Hence $(*)$ says that $N_{G}(B)^{\circ}$ centralizes $A_{0} / B$, in particular $A_{0}$ normalizes $C$, contradicting that $C$ is a Carter subgroup. Thus $B=B_{0}$ is the maximal $p$-unipotent subgroup of $N_{G}(B)^{\circ}$, so $B$ is a maximal $p$ unipotent subgroup of $G$.

Let $A$ be the largest normal (definable) subgroup of $G$ contained in $N_{G}(C)$. Since $A^{\circ} \leq N_{G}(C)^{\circ}=C$ is nilpotent, $A$ is necessarily finite.

By Fact 2.3, there is a characteristic $p$-divisible connected definable subgroup $U$ of $C$ such that $C=U * B$. Since $U$ is $p$-divisible and centralizes its $p$-torsion 
(Fact 2.3), and since $C$ has no nontrivial pseudo-torus, $U$ contains no $p$-element and $C=U \times B$. Moreover, by Fact 2.3, we have $H=(H \cap U) \times(H \cap B)$ for every definable subgroup $H$ of $C$. Since $C$ is not generous, $C \cap\left(\cup_{g \notin N_{G}(C)} C^{g}\right)$ is generic in $C$ and there exists $g \in G \backslash N_{G}(C)$ such that $C^{g} \cap C$ is not contained in $U A$. But we have $C^{g} \cap C=\left(C^{g} \cap U\right) \times\left(B^{g} \cap B\right)$, so $B \cap B^{g} \not \leq A$.

Since $A$ is finite, there exists $H=B \cap B_{0}$ an intersection between $B$ and a maximal $p$-unipotent subgroup $B_{0} \neq B$ of $G$, such that $H \not \leq A$ and such that, for every maximal $p$-unipotent subgroup $B_{1} \neq B$ of $G$, we have $r k\left(B_{1} \cap B\right) \leq r k(H)$. By Lemma 4.6, $N_{G}(H)^{\circ}$ is not solvable and it is a $C$-group since $H \not \leq A$ is not normal in $G$. Hence $N_{G}(H)^{\circ}$ contains a nontrivial pseudo-torus.

Let $B_{2}$ be a definable subgroup of $B$, not contained in $A$, such that $N_{G}\left(B_{2}\right)^{\circ}$ contains a nontrivial pseudo-torus and such that, for every definable subgroup $B_{3}$ of $B$ with $r k\left(B_{3}\right)>r k\left(B_{2}\right)$, the subgroup $N_{G}\left(B_{3}\right)^{\circ}$ contains no nontrivial pseudotorus. By the preceding paragraph, we have

$$
r k\left(B \cap B^{g}\right) \leq r k\left(B_{2}\right) \text { for every } g \notin N_{G}(B) .(* *)
$$

Since $N_{G}(B)^{\circ}$ has no nontrivial pseudo-torus by $(*)$ and Fact 2.22 , we have $B_{2}<$ $B$. Let $B_{3}=N_{B}\left(B_{2}\right)^{\circ}$ and $C_{2}=N_{C}\left(B_{2}\right)^{\circ}$. Then we have $r k\left(B_{3}\right)>r k\left(B_{2}\right)$ and $B_{3}=\left(B \cap C_{2}\right)^{\circ}$. Moreover, since $B_{2} \not \leq A, N_{G}\left(B_{2}\right)^{\circ} \neq G$ is a $C$-group and each Carter subgroup of $N_{G}\left(B_{2}\right)^{\circ}$ contains a nontrivial pseudo-torus by Fact

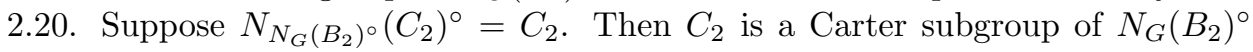
and $C_{2} \leq C$ contains a nontrivial pseudo-torus. This contradiction implies $C_{2}<$ $N_{N_{G}\left(B_{2}\right)^{\circ}}\left(C_{2}\right)^{\circ}$, in particular $N_{N_{G}\left(B_{2}\right)^{\circ}}\left(C_{2}\right)^{\circ}$ is not contained in $C$, and this proves that $N_{N_{G}\left(B_{2}\right)^{\circ}}\left(C_{2}\right)^{\circ}$ does not normalize $C$.

Let $h \in N_{N_{G}\left(B_{2}\right)^{\circ}}\left(C_{2}\right) \backslash N_{G}(C)$. Since $U \leq C_{C}(B)^{\circ}$, we have $C_{2}=U \times\left(B \cap C_{2}\right)$ and $B \cap C_{2}$ is connected. Hence $B \cap C_{2}=B_{3}$ and $U$ is definably characteristic in $C_{2}=U \times B_{3}$, in particular $h$ normalizes $U$. Since $h \notin N_{G}(C)$, we have $h \notin N_{G}(B)$ and $B \cap B^{h}<B$. But $h$ normalizes $C_{2}=U \times B_{3}$, therefore $h$ normalizes $B_{3}$ and we obtain $B_{2}<B_{3} \leq B \cap B^{h}<B$, contradicting (**).

Consequently $C$ is torsion-free.

Lemma 4.9 yields a link between Carter subgroups and $U_{0, r}$-subgroups.

Notation 4.8. - For every $G$ of finite Morley rank with a nontrivial torsion-free definable subgroup, let $\bar{r}_{\text {min }}(G)$ be the smallest integer such that $G$ has a nontrivial indecomposable subgroup $A$ of reduced rank $\bar{r}_{\min }(G)$.

Lemma 4.9. - Let $G$ be a $C$-group of finite Morley rank. If $G$ has a torsionfree Carter subgroup $C$ without a nontrivial pseudo-torus, then $G$ is a torsion-free solvable group, and $G$ has no nontrivial pseudo-torus.

Moreover, if $G \neq 1$, then $C$ contains a Sylow $U_{0, \bar{r}_{m i n}(G)}$-subgroup of $G$, In particular $\bar{r}_{\min }(G)=\bar{r}_{\min }(C)$.

Proof - Let $T$ be a maximal pseudo-torus of $G$. Then $T$ is contained in a Carter subgroup $D$ of $G$ (Fact 2.20) and, since $G$ is a $C$-group, $C$ contains a conjugate of $T$. Hence $T=1$ and $G$ is solvable. Moreover, if $G \neq 1$, then Facts 1.3 and 3.5 show

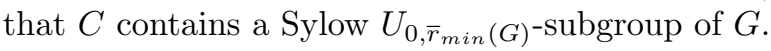

We assume toward a contradiction that $G$ has torsion. By Facts 2.1-2.4, there is a $G$-minimal $p$-unipotent subgroup $A$ in $G$ for a prime $p$. By Facts 2.6 and 2.8, the quotient $G / C_{G}(A)$ is a decent torus, and $G$ centralizes $A$ (Fact 2.22). In particular $C$ contains $A$, contradicting our hypothesis. 
Moreover, if $G \neq 1$, then Facts 1.3 and 3.5 show that $C$ contains a Sylow $U_{0, \bar{r}_{\text {min }}(G)}$-subgroup of $G$.

Proposition 4.10. - Let $G$ be a connected group of finite Morley rank, and $H$ a normal definable subgroup. If $H^{\circ}$ and $G / H$ are $C$-groups, then $G$ is a $C$-group.

Proof - We may suppose toward a contradiction that $G$ is a minimal counterexample. As $H$ and $G / H$ are $P$-groups, $G$ is a $P$-group, so $G$ has two non conjugate Carter subgroups, and $G$ has a non generous Carter subgroup $C$ (Fact 1.4).

Let $A$ be a normal subgroup of $G$ such that $G / A$ is a $C$-group. If $G \neq C A$, then $(C A)^{\circ}$ is a $C$-group by minimality of $G$, and the Carter subgroups of $C A$ are conjugate, therefore $N_{G / A}(C A / A)=N_{G}(C) A / A$ by a Frattini Argument. So $C A / A$ is a Carter subgroup of $G / A$, and $C A / A$ is generous in $G / A$ (Remark 4.5). Then, since $G$ centralizes the finite section $A / A^{\circ}$, the sugbroup $C A^{\circ}$ is generous in $G$. But $C$ is generous in the $C$-group $C A^{\circ}$, and this contradicts the nongenericity of $C$ in $G$ (Fact 2.11), hence we have $G=C A=C A^{\circ}$, in particular $G=C H^{\circ}$. Moreover this proves that $G$ has no infinite normal definable solvable subgroup $A$, otherwise $G / A$ would be a $C$-group by minimality of $G$ and $G=C A$ would be solvable, contradicting Fact 2.12.

Since $G$ is not a $C$-group and since $H^{\circ}$ is a $C$-group, there exists a maximal proper connected definable subgroup $M$ of $G$ containing $H^{\circ}$. Since $G / H=C H / H$ is nilpotent, $M H / H$ is normal in $G / H$, and $M=(M H)^{\circ}$ is normal in $G$. Moreover, by Proposition 4.7 and by minimality of $G, C$ is torsion-free and has no nontrivial pseudo-torus. We assume toward a contradiction that $C \cap M \neq 1$. Then there exists an integer $r_{0}$ such that $U_{0, r_{0}}(C \cap M)$ is not trivial. Let $r$ be the smallest integer such that $C$ normalizes a nontrivial nilpotent $U_{0, r}$-subgroup of $M$ and let $U$ be a maximal nilpotent $U_{0, r}$-subgroup of $M$ such that $C$ normalizes $U$. Let $N=N_{G}(U)^{\circ}$. Since $U$ is infinite and nilpotent, and since $G$ has no infinite normal definable solvable subgroup, we have $N \neq G$, and $N$ is a $C$-group by minimality of $G$. But $C$ is a torsion-free Carter subgroup of $N$ and $C$ has no nontrivial pseudotorus, so $N$ is a torsion-free solvable subgroup without a nontrivial pseudo-torus (Lemma 4.9).

Let $s=\bar{r}_{\min }(N \cap M)$ and let $S$ be a nontrivial $U_{0, s}$-subgroup of $N \cap M$. We show that $s=r$. We have $s \leq r$ and $E_{N}(S)$ contains a Carter subgroup of $N$ (Fact 2.15). By Fact 1.3, there exists $x \in N$ such that $C \leq E_{N}\left(S^{x}\right)$. Let $R=U_{0, s}\left(M \cap F\left(E_{N}\left(S^{x}\right)\right)\right)$. Since Fact 2.14 gives $S^{x} \leq R$, the minimality of $r$ yields $r=s$. Now $U R$ is a nilpotent $U_{0, r}$-subgroup of $M$ (Fact 3.6), and $U R$ is normalized by $C$, so $R \leq U$ by the maximality of $U$. This proves that $U$ contains a $N$-conjugate of each $U_{0, s}$-subgroup of $N \cap M$ and, by the normality of $U$ in $N, U$ is the unique Sylow $U_{0, r}$-subgroup of $N \cap M$. We obtain $U_{0, r}\left(N_{M}(U)\right)=U_{0, r}(N \cap M)=U$, so $U$ is a Sylow $U_{0, r}$-subgroup of $M$ (Fact 3.3). Moreover, $N$ is torsion-free, without a nontrivial pseudo-torus, and $r=s=\bar{r}_{\min }(N \cap M)$, therefore Fact 3.5 says that $U$ is contained in a Carter subgroup $C_{0}$ of $M$ as a normal subgroup. In particular we have $C_{0} \leq N$ and, as $N$ is torsion-free and without a nontrivial pseudo-torus, $C_{0}$ is a torsion-free Carter subgroup of the $C$-group $M$, and $C_{0}$ has no nontrivial pseudo-torus. Then Lemma 4.9 says que $M$ is solvable contradicting that $G$ has no infinite normal definable solvable subgroup. This proves that $C \cap M=1$ and, since $G=C H^{\circ}$, we obtain $H^{\circ}=M$. 
Consequently $C$ is a minimal nontrivial definable subgroup of $G$, in particular we have $C \cap C^{g}=1$ for each $g \in G \backslash N_{G}(C)$. Since $C$ is a Carter subgroup of $G$, this proves that $C$ is generous in $G$, contradicting our hypothesis over $C$.

Corollary 4.11. - Let $G$ be a connected P-group. Suppose that, for each definable connected section $H / K$ of $G$, either $H / K$ is a $C$-group, or $r k(H / K)=r k(G)$. If $G$ is not a $C$-group, then $Z(G)$ is finite and $G / Z(G)$ is a simple $C^{*}$-group.

Proof - By Proposition 4.10, $G$ has no proper infinite normal definable subgroup. So its proper normal definable subgroups are finite and central, in particular $Z(G)$ is finite and $G / Z(G)$ is a simple group (Fact 2.5). This proves that the proper connected definable sections of $G / Z(G)$ are $C$-sections, that $G / Z(G)$ has no nontrivial proper normal subgroup, and that, for any infinite abelian divisible definable subgroup $\bar{A}$ of $G / Z(G), N_{G / Z(G)}(\bar{A})^{\circ}$ is a $C$-group. Now it is enough to prove that $G / Z(G)$ has a non generous torsion-free Carter subgroup, with no nontrivial pseudo-torus.

By Proposition 4.10, $G / Z(G)$ is not a $C$-group. As $G / Z(G)$ is a $P$-group and as each proper connected definable section of $G / Z(G)$ are $C$-sections, $G / Z(G)$ has a non generous Carter subgroup (Fact 1.4). We conclude by Proposition 4.7.

Corollary 4.12. - Let $G$ be a group of finite Morley rank, $H$ a normal definable subgroup, and $C$ a Carter subgroup of $G$. If $H^{\circ}$ is a $C$-group, then $C H / H$ is a Carter subgroup of $G / H$ and all the Carter subgroups of $G / H$ have this form.

Proof - By Proposition 4.10, $C H^{\circ}$ is a $C$-group, so a Frattini argument applied with $C H$ gives $N_{G}(C H)=N_{G}(C) H$ and $C H / H$ is a Carter subgroup of $G / H$.

Now, if $D / H$ is a Carter subgroup of $G / H$, then $D^{\circ}$ is a $C$-group (Proposition 4.10 ). Let $E$ be a Carter subgroup of $D$. The preceding paragraph yields $D=E H$, so $N_{G}(E)^{\circ} \leq N_{G}(D)^{\circ}=D$, and $E$ is a Carter subgroup of $G$.

Lemma 4.13. - Let $G$ be a group of finite Morley rank. Suppose that $N_{G}(A)^{\circ}$ is a $C$-group for each nontrivial abelian divisible definable subgroup $A$. Then each non generous divisible Carter subgroup $C$ of $G$ is torsion-free, and has no nontrivial pseudo-torus.

ProOF - Let $T$ be the maximal pseudo-torus of $C$ (Fact 2.19). Suppose toward a contradiction that $T$ is nontrivial. Then $N_{G}(T)^{\circ}$ is a $C$-subgroup containing $C$, so $C$ is generous in $N_{G}(T)^{\circ}$. By Facts 2.11 and 2.21, we obtain a contradiction with the nongenerosity of $C$ in $G$.

Theorem 4.14. - Theorem 1.5 holds if and only if it holds for any $C^{*}$-group.

PROOF - If Theorem 1.5 holds, then it holds for any $C^{*}$-group, so we may assume that Theorem 1.5 holds for any $C^{*}$-group. We consider toward a contradiction a minimal counterexample $G$ to Theorem 1.5, with a center $Z=Z(G)$ of minimal degree. Then, by Fact 1.4, $G$ has a non generous divisible Carter subgroup $C$ and, by minimality, $G$ is connected.

We show that each connected definable $P$-section of $G$ is a $C$-section. Otherwise there is a minimal counterexample $H / K$ and, by Fact $1.3, H / K$ is not solvable. Thus $H / K$ has a nontrivial pseudo-torus $T_{H} / K$, and $T_{H} / K$ is contained in a Carter subgroup $C_{T} / K$ of $H / K$ (Fact 2.20). By Proposition 4.7, $C_{T} / K$ is generous in $H / K$. Let $Z_{H} / K=Z(H / K)$. By Corollary $4.11, Z_{H} / K$ is finite and $H / Z_{H}$ is a simple $C^{*}$-group. Now, since Theorem 1.5 holds for any $C^{*}$-group, $H / Z_{H}$ has 
no generous Carter subgroup. But Corollary 4.12 says that $C_{T} Z_{H} / Z_{H}$ is a Carter subgroup of $H / Z_{H}$, contradicting the generosity of $C_{T} / K$. This proves that each connected definable $P$-section of $G$ is a $C$-section.

By the preceding paragraph and by Lemma 4.13, $C$ is torsion-free, and has no nontrivial pseudo-torus. Now, since $G$ is not a $C^{*}$-group by the choice of $G, G$ has a nontrivial normal proper $P$-subgroup $N$ of maximal rank, in particular $N^{\circ}$ is a $C$-group.

We assume toward a contradiction that $N$ is infinite. Since $C N^{\circ}$ is a $C$-group by Proposition 4.10, $C N^{\circ}$ is a torsion-free solvable group (Lemma 4.9), and $N^{\circ}$ contains an abelian divisible $G$-minimal subgroup $A_{1}$. Then $G=N_{G}\left(A_{1}\right)$ is a connected $P$-group, so $G$ is a $C$-group by the second paragraph. This contradicts the existence of $C$, hence $N$ is finite, in particular $N$ is a nontrivial central subgroup of $G$. Moreover, by the maximality of the rank of $N$, the center $Z$ of $G$ is a nontrivial finite subgroup.

Since the preimage $Z_{2}(G)$ of $Z(G / Z(G))$ is a normal definable nilpotent subgroup of $G$, it is a normal $P$-subgroup of $G$, and $Z_{2}(G)$ is finite by the maximality of the rank of $N$, therefore $Z_{2}(G)$ is central in $G$, and $Z(G / Z)$ is trivial. Let $A / Z$ be a nontrivial abelian divisible definable subgroup of $G / Z$. Since $Z$ is finite, the subgroup $A^{\circ}$ is abelian, and divisible (Fact 2.3). Moreover, we have $A=A^{\circ} Z$, therefore $N_{G / Z}(A / Z)=N_{G}(A) / Z=N_{G}\left(A^{\circ}\right) Z / Z$ is a $P$-group. By the minimality of the degree of $Z$, Theorem 1.5 holds for $G / Z$, in particular its divisible Carter subgroups are conjugate.

Moreover, $C Z / Z$ is a divisible subgroup since $C$ is torsion-free, and $C Z / Z$ is non generous in $G / Z$ since $Z$ is finite. Therefore, since Corollary 4.12 says that $C Z / Z$ is a Carter subgroup of $G / Z$, Theorem 1.5 yields an interpretable field $K$ of characteristic zero such that $C Z / Z$ is a homogeneous $U_{K}$-subgroup, and it shows that $G / Z$ has no generous Carter subgroup. But, by Corollary 4.12 , for any generous Carter subgroup $C_{1}$ of $G$, the subgroup $C_{1} Z / Z$ is a Carter subgroup of $G / Z$, and $C_{1} Z / Z$ is generous by generosity of $C_{1}$, hence $G$ has no generous Carter subgroup. Since $C$ is torsion-free and since $Z$ is finite, we have $C \cap Z=1$, so $C \cong C Z / Z$ is a homogeneous $U_{K}$-subgroup. Consequently, by the choice of $G$, there is a non generous divisible Carter subgroup $D$ of $G$ non conjugate with $C$. But Corollary 4.12 says that $D Z / Z$ is a (divisible) Carter subgroup of $G / Z$, hence the preceding paragraph shows that $D Z / Z$ and $C Z / Z$ are conjugate. Now, since $Z$ is finite, we have $D=(D Z)^{\circ}$ and $C=(C Z)^{\circ}$, and $C$ and $D$ are conjugate. This is our final contradiction.

\section{About Corollaries 1.6 And 1.9}

In this section, we verify that Corollaries 1.6 and 1.9 are well consequences from Theorem 1.5.

The following definition is motivated by Corollary 3.17 .

Definition 5.1. - A torsion-free group $G$ of finite Morley rank is a $\widetilde{V}$-group if each indecomposable subgroup of $G$ is a $\widetilde{U}$-group.

Remark 5.2. - Any definable subgroup of a $\widetilde{V}$-group is a $\widetilde{V}$-group and, by Facts 3.1 and 3.16 , any definable quotient of a $\widetilde{V}$-group is a $\widetilde{V}$-group.

Any $\widetilde{V}$-group has no nontrivial pseudo-torus. 
Moreover, by Corollary 3.17, a nilpotent group $G$ of finite Morley rank is a $\widetilde{V}$-group if and only if it is a torsion-free $\widetilde{U}$-group.

Our first proof uses the following result.

Fact 5.3. - [1, Theorem 3] Let $G$ be a connected group of finite Morley rank whose Sylow p-subgroups are finite. Then $G$ contains no elements of order $p$.

Proof - (Corollary 1.6 follows from Theorem 1.5). Suppose toward a contradiction that $G$ is a minimal counterexample to Corollary 1.6, in particular $G$ is connected. By the minimality of $G$, for each connected definable $P$-section $H / K$ of $G$, either $H / K$ is a $C$-group or $r k(H / K)=r k(G)$. Then Proposition 4.7 says that any non generous Carter subgroup of $G$ is torsion-free, and its Carter subgroups are conjugate by Theorem 1.5 and Fact 1.4 .

We assume that $G$ has a non generous Carter subgroup $C$ and we show that $G$ is a $\widetilde{V}$-group. By Proposition 4.7, $C$ is torsion-free, and there is an interpretable field $K$ of characteristic zero such that $C$ is a homogeneous $U_{K}$-subgroup by Theorem 1.5 , in particular $C$ is a $\widetilde{U}$-subgroup.

First we prove that $G$ has no nontrivial pseudo-torus. Let $T$ be a maximal pseudo-torus of $G$. By Fact 2.20, there exists a Carter subgroup of $G$ containing $T$, and we may assume $T \leq C$ by the conjugacy of the Carter subgroups in $G$, so $T=1$ (Remark 3.9). Hence each connected $P$-subgroup of $G$ is solvable (Remark $4.3)$.

We assume toward a contradiction that $G$ has torsion. By the preceding paragraph and by Facts 2.1 and 5.3, there is a prime $p$ such that $G$ has a nontrivial maximal $p$-unipotent subgroup $B$. Let $D$ be a Carter subgroup of $N_{G}(B)$. Since $G$ has no nontrivial pseudo-torus, Facts 2.6, 2.8 and 2.22 show that $D$ centralizes each $D$-minimal section of $B$. Hence we have $B \leq E_{B D}(D)$, so $B D$ is nilpotent (Fact 2.14). This proves that $D=N_{N_{G}(B)}(D)^{\circ}$ contains $B$, therefore $B$ is the unique maximal $p$-unipotent subgroup of $D$ (Fact 2.3), and $N_{G}(D)$ normalizes $B$. Thus $D$ is a Carter subgroup of $G$, in particular $D$ is conjugate with $C$, contradicting that $C$ is torsion-free. This proves that $G$ is torsion-free.

Suppose toward a contradiction that $G$ is not a $\widetilde{V}$-group. Then $G$ has a definable nilpotent subgroup $C_{0}$ which is not a $\widetilde{U}$-group. We may assume that $r=\bar{r}_{\min }\left(C_{0}\right)$ is minimal for such a subgroup. Let $N$ be a definable nilpotent subgroup which is not a $\widetilde{U}$-group, and such that $r=\bar{r}_{\text {min }}(N)$. Then $N_{G}\left(U_{0, r}(N)\right)$ is a $P$-group and, by preceding paragraphs, it is a torsion-free solvable subgroup without a nontrivial pseudo-torus. In particular $E=E_{N_{G}\left(U_{0, r}(N)\right)}(N)$ is a definable subgroup containing a Carter subgroup $M$ of $N_{G}\left(U_{0, r}(N)\right.$ ) (Facts 2.14 and 2.15). By Fact 2.14, we have $N \leq F(E)$ and, by Lemma 3.20, we have $\widetilde{K}(N) \neq 1$. Then Corollary 3.21 and Lemma 3.24 say that $M$ is not a $\widetilde{U}$-subgroup. By minimality of $r$, we have $r \leq \bar{r}_{\min }(M)$, and Fact 3.6 shows that $U_{0, r}(N) M$ is nilpotent, therefore $M$ contains $U_{0, r}(N)$ and $r=\bar{r}_{\min }(M)$. Hence we can define a sequence $\left(C_{i}\right)_{i}$ of definable nilpotent subgroups of $G$, which are not $\widetilde{U}$-subgroups, such that $r=\bar{r}_{\min }\left(C_{i}\right)$ for each integer $i$, and where, for each $i, C_{i+1}$ is a Carter subgroup of $N_{G}\left(U_{0, r}\left(C_{i}\right)\right)$ contained in $E_{N_{G}\left(U_{0, r}\left(C_{i}\right)\right)}\left(C_{i}\right)$. Then the sequence $\left(U_{0, r}\left(C_{i}\right)\right)_{i}$ is increasing, and there exists an integer $k$ such that $U_{0, r}\left(C_{i}\right)=U_{0, r}\left(C_{k}\right)$ for any $i \geq k$. Now $N_{G}\left(C_{k+1}\right)$ normalizes $U_{0, r}\left(C_{k+1}\right)=U_{0, r}\left(C_{k}\right)$ and, since $C_{k+1}$ is a Carter subgroup of $N_{G}\left(U_{0, r}\left(C_{k}\right)\right)$, $C_{k+1}$ is a Carter subgroup of $G$. In particular $C_{k+1}$ is conjugate with $C$ (Theorem $1.5)$, contradicting that $C$ is a $\widetilde{U}$-subgroup. 
Proof - (Corollary 1.9 follows from Theorem 1.5). Since $A$ is simple, we have $C_{A}(A)=1$ and $C_{G}(A)$ is isomorphic to a subgroup of $G / A$, which is solvable. Moreover, if $L / C_{G}(A)$ is an abelian definable subgroup of $G / C_{G}(A)$, then either $A$ normalizes $L$, or $N_{G}(L)$ is solvable.

In the first case, we have $L \cap A=1$ since $A$ is simple, so $[A, L]=1$ and $L$ centralizes $A$, that is $L / C_{G}(A)=1$. Hence, by Corollary 1.6, the Carter subgroups of $G / C_{G}(A)$ are conjugate.

Let $C$ and $D$ be two Carter subgroups of $G$. We show that $C$ and $D$ are conjugate. Since $C_{G}(A)$ is solvable, its connected component is a $C$-group (Fact 1.3), and Corollary 4.12 allows to suppose $C C_{G}(A)=D C_{G}(A)$. Consequently $C$ and $D$ are two Carter subgroups of the solvable group $C C_{G}(A)$, so they are conjugate (Fact 1.3).

\section{The Bender method Revisited}

In a group of finite Morley rank, a Borel subgroup is a maximal solvable connected definable subgroup. In [6], J. Burdges studied the intersections between two Borel subgroups in minimal connected simple groups of finite Morley rank. In our context, we need to study the intersections between two Borel subgroups in a more general context.

From now on, we fix a $C^{*}$-group $G$, a non generous torsion-free Carter subgroup $C$ of $G$, with no nontrivial pseudo-torus, and let $r=\bar{r}_{m i n}(C)$. Moreover, by Fact 3.10 , we can fix an interpretable field $K$ of characteristic zero such that $r k(K)=r$ and $U_{K}(C) \neq 1$.

The following result is proven as in [6, Results 3.10, 3.18 and 3.23]. Also, by Fact 2.3, we removed the word connected from the sentence "every nilpotent connected subgroup of $H$ is abelian" [6, Proposition 4.1 (2)].

Fact 6.1. - (Compare with [6, Proposition 4.1]) Suppose that $G$ has a connected definable subgroup $F$ such that each $C$-subgroup of $G$ containing $F$ is solvable. If $F$ is contained in $H=\left(B_{1} \cap B_{2}\right)^{\circ}$ for two distinct Borel subgroups $B_{1}$ and $B_{2}$, then the following conditions hold:

- $H^{\prime}$ is a homogeneous $U_{L}$-subgroup for an interpretable field $L$ of characteristic 0 .

- Every nilpotent subgroup of $H$ is abelian.

- If $H$ is nonabelian, then $U_{L}\left(C_{G}(H)\right) \neq 1$.

By using Lemma 4.9, we obtain the following corollary.

Corollary 6.2. - If $C$ is contained in two distinct Borel subgroups $B_{1}$ and $B_{2}$, then $H=B_{1} \cap B_{2}$ is torsion-free and without a nontrivial pseudo-torus, and:

- $H^{\prime}$ is a homogeneous $U_{L}$-subgroup for an interpretable field $L$ of characteristic 0 .

- Every nilpotent subgroup of $H$ is abelian, in particular $C$ is abelian.

Concerning Fact 6.8 and [6, Proposition 5.9], we have a problem with the last paragraph of the proof of [6]. Indeed, in our context, it is not clear that $C_{G}^{\circ}(K)$ is solvable.

We will use, in this section, the following facts, which are similar to Fact 3.3, [6, Lemma 5.7] and Fact 3.4 respectively.

For every interpretable field $L$ of characteristic zero, a Sylow $U_{L}$-subgroup of a group $H$ of finite Morley rank is a maximal nilpotent $U_{L}$-subgroup of $H$. 
Fact 6.3. - (Compare with Fact 3.3) Let $H$ be a group of finite Morley rank, and $L$ an interpretable field of characteristic zero. Then the Sylow $U_{L}$-subgroups of $H$ are exactly those nilpotent $U_{L}$-subgroups $S$ such that $U_{L}\left(N_{H}(S)\right)=S$.

Fact 6.4. - (Compare with [6, Lemma 5.7]) Let $Q$ be a nilpotent group of finite Morley rank, without a nontrivial decent torus, and suppose $U_{L}(Q) \neq 1$ for an interpretable field $L$ of characteristic zero. Then there is a generic subset $Q_{L}^{*}$ of $Q$ such that $U_{L}(d(k)) \neq 1$ for all $k \in Q_{L}^{*}$.

Fact 6.5. - (Compare with Fact 3.4) Let $H$ be a solvable group of finite Morley rank. Then the Sylow $U_{L}$-subgroups of $H$ are conjugate for each interpretable field $L$ of characteristic zero.

Lemma 6.6. - $U_{K}(C)$ is a Sylow $U_{K}$-subgroup of $G$.

Proof - By Lemma 4.9, $N=N_{G}\left(U_{K}(C)\right)^{\circ}$ is solvable and $C$ contains a Sylow $U_{0, r}$-subgroup of $N$. But Fact 3.4 shows that each Sylow $U_{0, r}$-subgroup of $N$ contains a Sylow $U_{K}$-subgroup of $N$, hence $U_{K}(C)$ is a Sylow $U_{K}$-subgroup of $N$, and Fact 6.5 gives $U_{K}(N)=U_{K}(C)$. We conclude by Fact 6.3 .

Corollary 6.7. - Each C-subgroup $H$ of $G$ containing $U_{K}(C)$ is solvable.

Proof - Let $D$ be a Carter subgroup of $N_{H}\left(U_{K}(C)\right)$. Since $N_{G}\left(U_{K}(C)\right)$ contains $C$, Lemma 4.9 says that $N_{G}\left(U_{K}(C)\right)$ is a solvable torsion-free subgroup, without a nontrivial pseudo-torus, and that $\bar{r}_{\min }\left(N_{G}\left(U_{K}(C)\right)=r\right.$. Consequently $\bar{r}_{\text {min }}\left(N_{H}\left(U_{K}(C)\right)=r\right.$ and $D=\left\langle U_{0, s}(D) \mid s \geq r\right\rangle$, and Fact 3.6 proves that $D U_{K}(C)$ is nilpotent, so $D$ contains $U_{K}(C)$. But Lemma 6.6 says that $U_{K}(C)$ is a Sylow $U_{K}$-subgroup of $G$, therefore $U_{K}(C)$ is the unique Sylow $U_{K}$-subgroup of $D$ and $N_{H}(D)$ normalizes $U_{K}(C)$. This proves that $D$ is a Carter subgroup of $H$, and $H$ is solvable (Lemma 4.9).

Then we obtain the following fact, with a slightly different proof from the one of [6, Proposition 5.9].

Fact 6.8. - (Compare with [6, Proposition 5.9]) Either $C$ is abelian or $C$ is a homogeneous $U_{K}$-subgroup.

ProOF - Suppose toward a contradiction that $C$ is not abelian and that $C$ is not a homogeneous $U_{K}$-subgroup. By Fact 3.10, there exists another interpretable field $L \neq K$ of characteristic zero such that $U_{L}(C) \neq 1$. Since $C$ is non generous in $G$, its subset $X=C \cap \cup_{g \in G \backslash N_{G}(C)} C^{g}$ is generic in $C$. Moreover, by Corollary 6.2 , there is a unique Borel subgroup $B$ of $G$ containing $C$. Therefore, by Facts 2.12 and 6.4 , there is a generic subset $C^{*}$ of $X$ such that $C^{b} \cap C^{*}=\emptyset$ for each $b \in B \backslash N_{B}(C)$ and, for $i=K, L, U_{i}(d(c)) \neq 1$ for all $c \in C^{*}$.

If $U_{K}(C)$ is abelian, let $v \in C^{*}$ and let $V=U_{K}(d(v))$. Then there exists $g \in G \backslash N_{G}(C)$ such that $C^{g}$ contains $v$, and $C_{G}(V)^{\circ}$ is a $C$-subgroup containing $C$ and $C^{g}$ (Fact 3.10). By Corollary 6.7, $C_{G}(V)^{\circ}$ is solvable, so it is contained in $B$, in particular $B$ contains $C^{g}$ and there exists $b \in B$ such that $C^{g}=C^{b}$ (Fact 1.3). This contradicts our choice of $v \in C^{*}$, and $U_{K}(C)$ is not abelian.

Now Fact 6.1 and Corollary 6.7 say that each $C$-subgroup of $G$ containing $U_{K}(C)$ is contained in $B$. Let $w \in C^{*}$ and let $W=U_{L}(d(w))$. Then there exists $h \in G \backslash N_{G}(C)$ such that $C^{h}$ contains $w$, and $C_{G}(W)^{\circ}$ is a $C$-subgroup containing $U_{K}(C)$ and $U_{K}\left(C^{h}\right)$ (Fact 3.10). Hence we have $U_{K}(C) \leq\left(C_{G}(W)^{\circ}\right)^{h^{-1}}$, so 
$\left(C_{G}(W)^{\circ}\right)^{h^{-1}} \leq B$ and $U_{K}(C)$ is contained in $C_{G}(W)^{\circ} \leq B^{h}$. This proves that $B^{h}=B$ and there exists $b \in B$ such that $C^{h}=C^{b}$ (Fact 1.3 ). This contradicts our choice of $w \in C^{*}$.

Also, the following fact will be very important for us. Its proof is similar to the one of [6, Theorem 5.1].

Fact 6.9. - (Compare with [6, Theorem 5.1]) If $G$ has a $C$-subgroup $H$ containing strictly $C$, then $H$ is contained in a unique Borel subgroup of $G$.

\section{Structure of nON Generous Carter subgroups}

In this section we prove that $C$ is a $\widetilde{V}$-group (Definition 5.1 , Theorem 7.3 ), that is a weak form of the second part of Theorem 1.5. This result is fundamental for us, and its proof, which uses Fact 2.9 and Proposition 7.2, is the least trivial proof of this paper.

Note that, by Lemma 7.1, we will have another important structural result: any proper definable connected subgroup of $G$ containing $C$ is a solvable $\widetilde{V}$-group.

Lemma 7.1. - Let $H$ be a $C$-group and $D$ a Carter subgroup of $H$. If $D$ is a $\widetilde{V}$-group, then $H$ is a solvable $\widetilde{V}$-group.

Proof - By Lemma 4.9, $H$ is a torsion-free solvable group, and $H$ has no nontrivial pseudo-torus. Let $A$ be an indecomposable subgroup of $H$. By Facts 2.15 and 1.3, there exists $x \in H$ such that $D^{x} \leq E_{H}(A)$. By Fact 2.14 and Corollary 3.21 , we have $\widetilde{K}(A) \leq \widetilde{K}\left(F\left(E_{H}(A)\right)\right)$, and Lemma 3.24 gives $\widetilde{K}(A) \leq \widetilde{K}\left(D^{x}\right)$. Since $D$ is a $\widetilde{U}$-group, we have $\widetilde{K}\left(D^{x}\right)=1$, therefore $\widetilde{K}(A)=1$ and Lemma 3.20 proves that $A$ is a $\widetilde{U}$-group.

Proposition 7.2. - Let $H$ be a torsion-free solvable group of finite Morley rank without a nontrivial pseudo-torus, and $D$ be a Carter subgroup of $H$. Let $V$ be a definable subgroup of $D, X$ a nonempty definable subset of $H$ and $\mathscr{F}=\left\{V \cap D^{x} \mid x \in\right.$ $X\}$. If $\cup \mathscr{F}$ is generic in $V$, then there exists $x \in X$ such that $D^{x}$ contains $\widetilde{K}(V)$.

ProOF - We proceed by induction on $r k(H)$. If $F(H)$ contains $V$, then Corollary 3.21 and Lemma 3.24 yield $\widetilde{K}(V) \leq \widetilde{K}(F(H)) \leq \widetilde{K}\left(D^{x}\right)$ for each $x \in X$. Hence we may assume $V \not \leq F(H)$.

Let $U$ be a normal definable subgroup of $H$, maximal among the ones such that $V U / U \not \leq F(H / U)$. Let $F$ be the preimage of $F(H / U)$ in $H$. By Fact 2.14 we have $E_{H}(V U / U) \neq H / U$, and Fact 2.15 gives $H \neq U E_{H}(V)$. Let $A / U$ be a $H$-minimal subgroup of $H / U$. By maximality of $U$ we obtain $V A / A \leq F(H / A)$ and Fact 2.15 yields $H=A E_{H}(V)$, in particular we have $F=A\left(F \cap E_{H}(V)\right)$.

Thus, if $F \neq A$, then $\left(F \cap E_{H}(V)\right) U / U$ is nontrivial and contains an $E_{H}(V)$ minimal subgroup $B / U$. Since $B \leq F$ centralizes $A / U, B$ is normal in $H=$ $A E_{H}(V)$. By maximality of $U$, we obtain $V B / B \leq F(H / B)$, and Facts 2.14 and 2.15 yield $H=B E_{H}(V)$. But $B$ is contained in $U E_{H}(V)$, hence $H=U E_{H}(V)$, contradicting the preceding paragraph. Consequently we have $F=A$.

By Facts 2.4 and 2.13, we have $H=A D$, in particular $H$ normalizes $(A \cap D) U / U$ and, by $H$-minimality of $A / U$, either $H=U D$ or $H / U=A / U \rtimes D U / U$. But we have $H \neq U E_{H}(V)$, and $D \leq E_{H}(V)$ since $D$ is nilpotent and contains $V$, therefore $H \neq U D$ and $H / U=A / U \rtimes D U / U$. 
As $V \not \leq U$, there exists $g \in X$ such that $W=V \cap D^{g}$ is not contained in $U$. Then $W$ is not contained in $A=F$ and we have $W \leq E_{H}(W)$, in particular $H=A E_{H}(W)$. By Facts 2.14 and 2.15, we have $H \neq U E_{H}(W)$ and, by $H$ minimality of $A / U$, we obtain $A \cap E_{H}(W) \leq U$. Consequently $E_{H}(W) U / U$ is a complement of $A / U$ in $H / U$, and $E_{H}(W) U / U$ contains $D U / U$, so $E_{H}(W) U=D U$. Since $E_{H}(W)$ contains $D^{g}$, we obtain $D^{g} \leq D U$ and Fact 2.13 gives $g \in D U$.

Let $X^{*}=\left\{x \in X \mid V \cap D^{x} \not \leq U\right\}$ and $\mathscr{F}^{*}=\left\{V \cap D^{x} \mid x \in X^{*}\right\}$. By the preceding paragraph, we have $X^{*} \subseteq D U$. Since $\cup\left(\mathscr{F} \backslash \mathscr{F}^{*}\right) \subseteq U$ and $V \not \leq U$, the subset $\cup \mathscr{F}^{*}$ is generic in $V$. But we have $H / U=A / U \rtimes D U / U$, hence $D U<H$, and the induction hypothesis applied to $D U$ yields $x \in X^{*} \subseteq X$ such that $\widetilde{K}(V) \leq D^{x}$. This proves the result.

Theorem 7.3. - $C$ is a $\widetilde{V}$-subgroup.

Proof - We assume toward a contradiction that $C$ is not a $\widetilde{V}$-group. By Corollary 3.17, $C$ is not a $\widetilde{U}$-subgroup and, by Fact $6.8, C$ is abelian. Let $C_{X}=$ $C \cap\left(\cup_{g \notin N_{G}(C)} C^{g}\right)$. As $C$ is non generous, we have

$$
\begin{aligned}
r k\left(C \backslash C_{X}\right)-r k(C)+r k(G) & =r k\left(C \backslash C_{X}\right)+r k\left(G / N_{G}(C)\right) \\
& \leq r k\left(\cup_{g \in G} C^{g}\right) \\
& <r k(G)
\end{aligned}
$$

therefore $C_{X}$ is generic in $C$. We consider $X_{C}=\left\{x \in G \mid \widetilde{K}(C) \not \subset C^{x}\right\}, X_{C}^{\prime}=$ $G \backslash\left(N_{G}(C) \cup X_{C}\right), \mathscr{F}_{C}=\left\{C \cap C^{x} \mid x \in X_{C}\right\}$ and $\mathscr{F}_{C}^{\prime}=\left\{C \cap C^{x} \mid x \in X_{C}^{\prime}\right\}$. Then we have $C_{X}=\left(\cup \mathscr{F}_{C}\right) \cup\left(\cup \mathscr{F}_{C}^{\prime}\right)$, so either $\cup \mathscr{F}_{C}$ is generic in $C$ or $\cup \mathscr{F}_{C}^{\prime}$ is generic in $C$.

We prove that $\cup \mathscr{F}_{C}^{\prime}$ is not generic in $C$. Since $C$ is not a $\widetilde{U}$-group, we have $\widetilde{K}(C) \neq 1$ (Lemma 3.20) and $C_{0}=C_{G}(\widetilde{K}(C))^{\circ}$ is a $C$-group. As $C$ is abelian, $C_{0}$ contains $C$ and Lemma 4.9 says that $C_{0}$ is solvable. By Fact 2.12, there is a definable non generic subset $J$ of $C$ such that, for each $g \in C_{0} \backslash N_{G}(C), C \cap C^{g}$ is contained in $J$. For every $x \in X_{C}^{\prime}$, we have $C^{x} \leq C_{0}$ since $C$ is abelian, so Fact 1.3 gives $y \in C_{0}$ such that $C^{x}=C^{y}$. Thus we have $\mathscr{F}_{C}^{\prime}=\left\{C \cap C^{x} \mid x \in X_{C}^{\prime} \cap C_{0}\right\}$ and $\cup \mathscr{F}_{C}^{\prime} \subseteq J$, in particular $\cup \mathscr{F}_{C}^{\prime}$ is not generic in $C$. By the preceding paragraph, $\cup \mathscr{F}_{C}$ is generic in $C$.

We consider a definable subgroup $V$ of $C$, minimal among the ones such that $\cup \mathscr{F}_{V}$ is generic in $V$, where $\mathscr{F}_{V}=\left\{V \cap C^{x} \mid x \in X_{V}\right\}$ and $X_{V}=\{x \in G \mid \widetilde{K}(V) \not \leq$ $\left.C^{x}\right\}$. In particular $V$ is not a $\widetilde{U}$-group. By Fact 2.9 , there is an algebraically closed field $L$ and a normal definable subgroup $N$ of $V$ such that $V / N$ is definably isomorphic to a $L$-vector space of dimension 2 , and we may consider $V / N$ as a $L$-vector space.

We assume toward a contradiction that $N$ is not a $\widetilde{U}$-group, that is $\widetilde{K}(N) \neq 1$ (Lemma 3.20). Let $\mathscr{F}_{K N}=\left\{F \in \mathscr{F}_{V} \mid \widetilde{K}(N) \not \leq F\right\}$. Suppose that $\cup \mathscr{F}_{K N}$ is generic in $V$. Then there is a vector subspace $W / N$ of dimension 1 of $V / N$ such that $\left(\cup \mathscr{F}_{K N}\right) \cap W$ is generic in $W$. If $X_{K N}=\left\{x \in G \mid V \cap C^{x} \in \mathscr{F}_{K N}\right\}$, then $\cup\left\{W \cap C^{x} \mid x \in X_{K N}\right\}=\left(\cup \mathscr{F}_{K N}\right) \cap W$ is generic in $W$ and, by the minimality of $V$, there is $x \in X_{K N}$ such that $C^{x}$ contains $\widetilde{K}(W)$. By Corollary 3.21 we obtain $\widetilde{K}(N) \leq \widetilde{K}(W) \leq C^{x} \cap W \leq C^{x} \cap V \in \mathscr{F}_{K N}$ and this contradiction proves that $\cup \mathscr{F}_{K N}$ is not generic in $V$. Thus, if $\mathscr{F}_{N}=\left\{F \in \mathscr{F}_{V} \mid \widetilde{K}(N) \leq F\right\}$, then $\cup \mathscr{F}_{N}$ is generic in $V$. As $C$ is abelian, the subgroup $C_{G}(\widetilde{K}(N))^{\circ}$ contains $C$ and, by 
Lemma 4.9 , it is solvable. Moreover, for each $x \in X_{V}$ such that $V \cap C^{x} \in \mathscr{F}_{N}$, we have $\widetilde{K}(N) \leq C^{x}$, so $C^{x} \leq C_{G}(\widetilde{K}(N))^{\circ}$ and there is $y \in C_{G}(\widetilde{K}(N))^{\circ}$ such that $C^{x}=C^{y}$ (Fact 1.3). This proves that $\cup\left\{V \cap C^{y} \mid y \in X_{V} \cap C_{G}(\widetilde{K}(N))^{\circ}\right\}$ is a generic subset of $V$ and Proposition 7.2 shows that there is $y \in X_{V} \cap C_{G}(\widetilde{K}(N))^{\circ}$ such that $\widetilde{K}(V) \leq C^{y}$, contradicting the choice of $X_{V}$. This proves that $N$ is a $\widetilde{U}$-group.

By Corollary 3.17, $V$ has an indecomposable subgroup $A$ which is not a $\widetilde{U}$-group. We show that

$\left(\cup\left\{E \in \mathscr{F}_{V} \mid E A \neq V\right\}\right) A$ is not generic in $V$ for such any subgroup $A$. (*)

Indeed, if $A$ is an indecomposable subgroup of $V$ which is not a $\widetilde{U}$-group, if $\mathscr{F}_{A}=$ $\left\{E \in \mathscr{F}_{V} \mid E A \neq V\right\}$ and if $\left(\cup \mathscr{F}_{A}\right) A$ is generic in $V$, then Fact 2.9 yields an algebraically closed field $K_{1}$ and a normal definable subgroup $M / A$ of $V / A$ such that $V / M$ is definably isomorphic to a $K_{1}$-vector space of dimension 2 . But the preceding paragraph with $N=M$ proves that $M$ is a $\widetilde{U}$-group, contradicting $A \leq M$ (Fact 3.16).

By Fact 2.7, for each definable subgroup $F$ of $V$, the subgroup $F N / N$ is a vector subspace of $V / N$. Let $\mathscr{F}_{V 1}=\left\{F \in \mathscr{F}_{V} \mid \operatorname{dim}(F N / N)=1\right\}$. We show that $\cup \mathscr{F}_{V 1}$ is generic in $V$. For each $F \in \mathscr{F}_{V}$, if $\operatorname{dim}(F N / N)=2$, then $V=F N$ and $V / \widetilde{K}(F)=(F / \widetilde{K}(F))(N \widetilde{K}(F) / \widetilde{K}(F))$ is a $\widetilde{U}$-group (Lemma 3.20 and Facts 3.14 and 3.16). Hence we have $\widetilde{K}(V) \leq \widetilde{K}(F) \leq F$, contradicting $F \in \mathscr{F}_{V}$. As the union $\cup\left\{F \in \mathscr{F}_{V} \mid \operatorname{dim}(F N / N)=0\right\}$ is contained in $N$ and is not generic in $V$, and as $\cup \mathscr{F}_{V}$ is generic in $V$, we obtain the genericity of $\cup \mathscr{F}_{V 1}$ in $V$.

We consider a definable subset $X_{V}^{*}$ of $X_{V 1}=\left\{x \in G \mid V \cap C^{x} \in \mathscr{F}_{V 1}\right\} \subseteq X_{V}$ of degree 1 and of minimal Morley rank among the ones such that $\cup\left\{V \cap C^{x} \mid x \in X_{V}^{*}\right\}$ is generic in $V$. Let $\mathscr{F}_{V}^{*}=\left\{V \cap C^{x} \mid x \in X_{V}^{*}\right\}$. For each $F \in \mathscr{F}_{V}^{*}$, there exists $F_{0} \in \mathscr{F}_{V}^{*}$ such that $F N \neq F_{0} N$ since $\operatorname{dim}(F N / N)=1$ and since $\cup \mathscr{F}_{V}^{*}$ is generic in $V$. So we have $\operatorname{dim}\left(F F_{0} N / N\right)=2$ and $V=F F_{0} N$, and we obtain $V / \widetilde{K}(F) \widetilde{K}\left(F_{0}\right)=$ $F F_{0} N / \widetilde{K}(F) \widetilde{K}\left(F_{0}\right)$, in particular $V / \widetilde{K}(F) \widetilde{K}\left(F_{0}\right)$ is a $\widetilde{U}$-group (Lemma 3.20 and Facts 3.14 and 3.16). Thus, by Corollary 3.21 , we have $\widetilde{K}(V)=\widetilde{K}(F) \widetilde{K}\left(F_{0}\right)$. As $F_{0} \in \mathscr{F}_{V}^{*} \subseteq \mathscr{F}_{V}$, we have $\widetilde{K}(V) \not \leq F_{0}$ and $\widetilde{K}(V) \neq \widetilde{K}\left(F_{0}\right)$, in particular $\widetilde{K}(F)$ is nontrivial.

Let $F$ be a fixed $\mathscr{F}_{V}^{*}$-subgroup, and $x \in G$ satisfying $F=V \cap C^{x}$. By Lemma 4.9 and since $F \in \mathscr{F}_{V}^{*}$ implies $C^{x} \neq C$, the subgroup $U=\left\langle C, C^{x}\right\rangle$ of $C_{G}(\widetilde{K}(F))^{\circ}$ is solvable and nonnilpotent. By Corollary 3.17, $F$ has an indecomposable subgroup $A$ which is not a $\widetilde{U}$-group. By $(*)$, if $\mathscr{F}_{F}^{*}=\left\{E \in \mathscr{F}_{V}^{*} \mid E A=V\right\}$, the subset $\cup \mathscr{F}_{F}^{*}$ is generic in $V$.

Let $\mathscr{F}_{0}^{*}=\left\{E \in \mathscr{F}_{F}^{*} \mid E \cap F \neq 1\right\}$. We assume toward a contradiction that $\cup \mathscr{F}_{0}^{*}$ is a generic subset of $V$. By Fact 6.9, the subgroup $U$ is contained in a unique Borel subgroup $B_{U}$ of $G$. For each $E \in \mathscr{F}_{0}^{*}$ and each $x_{E} \in G$ such that $E=V \cap C^{x_{E}}$, as $E \cap F$ is nontrivial, the subgroup $C_{G}(E \cap F)^{\circ}$ is solvable (Lemma 4.9) and contains $U$ and $C^{x_{E}}$. So we have $C^{x_{E}} \leq C_{G}(E \cap F)^{\circ} \leq B_{U}$ for each such an element $x_{E}$. By Fact 1.3 , for each $E \in \mathscr{F}_{0}^{*}$, there exists $y_{E} \in B_{U}$ such that $E=V \cap C^{y_{E}}$. If we consider $X_{0}^{*}=\left\{x \in B_{U} \mid V \cap C^{x} \in \mathscr{F}_{0}^{*}\right\}$, then Proposition 7.2 yields a contradiction and this proves that $\cup \mathscr{F}_{0}^{*}$ is not generic in $V$.

Let $\mathscr{F}_{F}=\cup \mathscr{F}_{F}^{*} \backslash \cup \mathscr{F}_{0}^{*}$. By the preceding paragraph, the union $\cup \mathscr{F}_{F}$ is generic in $V$. In the same way, if we consider $E \in \mathscr{F}_{F}$, then $E$ has an indecomposable 
subgroup $B$ which is not a $\widetilde{U}$-group, and the union $\cup \mathscr{F}_{E}$ is generic in $V$ where $\mathscr{F}_{E}=\left\{E_{0} \in \cup \mathscr{F}_{V}^{*} \mid E_{0} B=V\right.$ and $\left.E_{0} \cap E=1\right\}$. By the choice of $X_{V}^{*}$ and $\mathscr{F}_{V}^{*}$, the union $\cup\left(\mathscr{F}_{E} \cap \mathscr{F}_{F}\right)$ is generic in $V$. In particular there exists $M \in \mathscr{F}_{E} \cap \mathscr{F}_{F}$ and, thus, we have $M \cap E=M \cap F=1, V=M B \leq M E \leq V$ and $V=M A \leq M F \leq V$, consequently

$$
E \cong E M / M=V / M=F M / M \cong F
$$

Moreover, we have $E=E \cap M B=(E \cap M) B=B$ and $F=A$, so $E$ and $F$ are indecomposable subgroups of $V$. Since $\operatorname{dim}(F N / N)=\operatorname{dim}(E N / N)=1$, the subgroup $F \cap N$ (resp. $E \cap N$ ) is maximal in $F$ (resp. $E$ ) by Fact 2.7, and we obtain $J(F)=F \cap N$ and $J(E)=E \cap N$, in particular $J(E) J(F) \leq N$.

Since $E \in \mathscr{F}_{F}$, we have $E F=E A=V$ and, as $\operatorname{dim}(E N / N)=1=\operatorname{dim}(F N / N)$ and $\operatorname{dim}(V / N)=2$, we have $E N \cap F N=N$, in particular $E \cap F N \leq N$. Also we have $J(E) F \leq N F<V$ and, by the maximality of $J(E)$ in $E$, the subgroup $J(E) F$ is maximal in $E F=V$, hence $J(E) F=N F$ and

$$
N=N \cap N F=N \cap J(E) F=J(E)(N \cap F)=J(E) J(F) .
$$

Let $Y / N$ be a vector subspace of dimension 1 of $V / N$ such that $\cup\left(\mathscr{F}_{E} \cap \mathscr{F}_{F}\right) \cap Y$ is generic in $Y$. Then the union of the subgroups $P \cap Y$ where $P \in \mathscr{F}_{E} \cap \mathscr{F}_{F}$ and $P \cap(Y \backslash N) \neq \emptyset$ is generic in $Y$. But, for such any subgroup $P$, we have $P N / N \cap Y / N \neq 1$ and, as $\operatorname{dim}(P N / N)=1=\operatorname{dim}(Y / N)$, we obtain $Y=P N$ and $P \leq Y$. Hence, if $\mathscr{F}_{Y}=\left\{P \in \mathscr{F}_{E} \cap \mathscr{F}_{F} \mid P \leq Y\right\}$, the union $\cup \mathscr{F}_{Y}$ is generic in $Y$. Moreover, in the same way than for $E$, every $P \in \mathscr{F}_{Y}$ is definably isomorphic to $F$, in particular $P$ is indecomposable. Moreover, since $F / J(F) \cong F N / N$ is definably isomorphic to a vector subspace of dimension 1 of $V / N$, we have $F=U_{L}(F)$ and every $P \in \mathscr{F}_{Y}$ is a $U_{L}$-subgroup, so $Y$ is a $U_{L}$-subgroup. Let $P$ be a fixed $\mathscr{F}_{Y^{-}}$ subgroup. Then we have $V=P F$ and $Y=Y \cap P F=(Y \cap F) P$ and, as $P \cap F=1$, the subgroup $Y \cap F$ is a $U_{L}$-subgroup (Fact 3.18). Since $F /(Y \cap F) \cong F Y / Y=V / Y$ is definably isomorphic to a quotient space of dimension 1 of $V / N$, the subgroup $Y \cap F$ is maximal in $F$ (Fact 2.7) and $J(F)=Y \cap F$, so $J(F)$ is a $U_{L}$-subgroup. In the same way, $J(E)$ is a $U_{L}$-subgroup, therefore $N=J(E) J(F)$ is a $U_{L}$-subgroup. But $N$ is a $\widetilde{U}$-group, so $N$ is a homogeneous $U_{L}$-subgroup (Fact 3.13). Now $F$ is a $U_{L}$-subgroup and each proper subgroup of $F$ is contained in $J(F) \leq N$, which is a homogeneous $U_{L}$-subgroup, so $F$ is a homogeneous $U_{L}$-subgroup and $F$ is a $\widetilde{U}$-group. In the same way $E$ is a $\widetilde{U}$-group and $V=E F$ is a $\widetilde{U}$-group too (Fact $3.14)$.

\section{Intersections of CARTER Subgroups}

In this section we study the conjugacy of Carter subgroups with a nontrivial intersection between them.

We recall that $C$ is a $\widetilde{V}$-group, and a $\widetilde{U}$-group by Theorem 7.3 and Corollary 3.17. Moreover, by Fact $3.13, U_{K}(C)$ is a homogeneous $U_{K}$-subgroup.

Lemma 8.1. - For each nontrivial definable subgroup $U$ of $C$, the following conditions hold:

1. $N_{G}(U)^{\circ}$ is a solvable $\widetilde{V}$-group;

2. $\bar{r}_{\min }\left(N_{G}(U)\right)=r$;

3. any Sylow $U_{K}$-subgroup of $N_{G}(U)$ is conjugate to a subgroup of $U_{K}(C)$. 
ProOF - If $U$ is normal in $C$, then Lemmas 7.1 and 4.9 and Fact 3.4 give the result, so we may assume that $U$ is not normal in $C$, in particular $C$ is nonabelian and $U \neq C$. Also, by Fact $6.8, C$ is a homogeneous $U_{K}$-subgroup. We may assume that, for every definable subgroup $U_{0}$ of $C, r k(U)<r k\left(U_{0}\right)$ implies: $1 . N_{G}\left(U_{0}\right)^{\circ}$ is a solvable $\widetilde{V}$-group; 2. $\bar{r}_{\min }\left(N_{G}\left(U_{0}\right)\right)=r ; 3$. any Sylow $U_{K^{-}}$subgroup of $N_{G}\left(U_{0}\right)$ is conjugate to a subgroup of $U_{K}(C)$.

Let $N=N_{G}(U)^{\circ}$. We may assume that, for each $g \in G$, we have either $U \not \leq C^{g}$ or $r k\left(N \cap C^{g}\right) \leq r k(N \cap C)$. Let $V=N_{C}(U)=N \cap C$. Then we have $r k(U)<$ $r k(V)$, consequently $1 . N_{G}(V)^{\circ}$ is a solvable $\widetilde{V}$-group; $2 . \bar{r}_{\min }\left(N_{G}(V)\right)=r ; 3$. any Sylow $U_{K}$-subgroup of $N_{G}(V)$ is conjugate to a subgroup of $C$.

Now, if $L$ is a Carter subgroup of $N_{N}(V)$, then $L$ is a nilpotent $\widetilde{V}$-subgroup, $\bar{r}_{\text {min }}(L) \geq r$ and there exists $g \in G$ such that $U_{K}(L) \leq C^{g}$. By Fact 3.6, the subgroup $L V$ is nilpotent, therefore $L$ contains $V$, and we have $U<V \leq U_{K}(L) \leq$ $N \cap C^{g}$. Since $V=N \cap C$ and since $U \leq C^{g}$, we have $r k\left(N \cap C^{g}\right) \leq r k(N \cap C)$, and we obtain $V=N \cap C=U_{K}(L)=N \cap \bar{C}^{g}$. Since $N_{G}(V)^{\circ}$ is a solvable $\widetilde{V}$-group, it is a $C$-group with no nontrivial pseudo-torus and, since $\bar{r}_{\min }\left(N_{G}(V)\right)=r$, Lemma 4.9 says that $U_{0, r}(L)$ is a Sylow $U_{0, r}$-subgroup of $N_{N}(V)$. Moreover, since $r k(K)=r$, Fact 3.4 shows that each Sylow $U_{K}$-subgroup of $N_{N}(V)$ is conjugate to $U_{K}\left(U_{0, r}(L)\right)$ hence, since $r k(K)=r$ implies $U_{K}\left(U_{0, r}(L)\right)=U_{K}(L)=V$, the subgroup $V$ is the unique Sylow $U_{K}$-subgroup of $N_{N}(V)$. Thus we obtain $V=U_{K}\left(N_{N}(V)\right)$ and Fact 6.3 says that $V$ is a Sylow $U_{K}$-subgroup of $N$.

Since $V=U_{K}(L)$, the subgroup $N_{N}(L)$ normalizes $V$ and we have $N_{N}(L)^{\circ}=$ $N_{N_{N}(V)}(L)^{\circ}=L$, so $L$ is a Carter subgroup of $N$. Consequently, by Lemma 7.1, $N$ is a solvable $\widetilde{V}$-group and, by Lemma $4.9, \bar{r}_{\min }(N)=\bar{r}_{\min }(L)=r$. Moreover, if $S$ is a Sylow $U_{K}$-subgroup of $N_{G}(U)$, then Fact 6.5 yields $x \in G$ such that $V \leq C$ contains $S^{x}$.

From now on, we obtain the following improvement of Corollary 6.7.

Proposition 8.2. - Let $B$ be a $C$-subgroup of $G$. If $B \cap U_{K}(C) \neq 1$, then $B$ is a solvable $\widetilde{V}$-group and $\bar{r}_{\min }(B)=r$.

Proof - We assume toward a contradiction that $G$ has a $C$-subgroup $B$ such that $B \cap U \neq 1$, where $U=U_{K}(C)$, and that either $B$ is not a solvable $\widetilde{V}$-group or $\bar{r}_{\text {min }}(B)<r$. We may assume that, for every $C$-subgroup $B_{0}$ of $G$, if $r k(B \cap U)<$ $r k\left(B_{0} \cap U\right)$, then $B_{0}$ is a solvable $\widetilde{V}$-group and $\bar{r}_{\text {min }}\left(B_{0}\right)=r$. Let $H=B \cap U$ and $N=N_{B}(H)^{\circ}$. By Lemma $8.11 ., N$ is a solvable $\widetilde{V}$-group and, by Lemmas 4.9 and 8.12 ., $U_{0, r}(D)$ is a Sylow $U_{0, r}$-subgroup of $N$ for each Carter subgroup $D$ of $N$, and $\bar{r}_{\min }(D)=r$. Moreover, Fact 3.6 shows that $D H$ is nilpotent, so $D$ contains $H$, hence $H \leq U_{K}(D)$. Now, since Lemma 8.1 3. provides $g \in G$ such that $U_{K}(D)^{g} \leq U$, we obtain

$$
r k(B \cap U)=r k(H) \leq r k\left(U_{K}(D)\right)=r k\left(U_{K}(D)^{g}\right) \leq r k\left(B^{g} \cap U\right) .
$$

Consequently we have $r k(B \cap U)=r k\left(B^{g} \cap U\right)$ and $H=U_{K}(D)$, in particular $H$ is normal in $N_{B}(D)$ and $N_{B}(D)^{\circ} \leq N_{B}(H)^{\circ}=N$. Thus $N_{B}(D)^{\circ}=N_{N}(D)^{\circ}=D$ and $D$ is a Carter subgroup of $B$.

Since $N$ is a solvable $\widetilde{V}$-group, $D$ is a $\widetilde{V}$-group and $B$ is a solvable $\widetilde{V}$-group (Lemma 7.1). Now Lemma 4.9 yields $\bar{r}_{\min }(B)=r$ and a contradiction. 
Corollary 8.3. - Let $U$ be a nonabelian subgroup of $C$. Then $U$ is contained in a unique maximal $C$-subgroup $B$ of $G$. Moreover, $B$ is a solvable $\widetilde{V}$-group.

Proof - By Fact 6.8, $C$ is a homogeneous $U_{K}$-subgroup of $G$. By Proposition 8.2, every $C$-subgroup of $G$ containing $U$ is a solvable $\widetilde{V}$-subgroup, and Fact 6.1 yields the uniqueness of $B$.

Proposition 8.4. - Each Sylow $U_{K}$-subgroup $S$ of $G$ such that $S \cap C \neq 1$ is conjugate with $U_{K}(C)$.

Proof - By Lemma 6.6, $U=U_{K}(C)$ is a Sylow $U_{K}$-subgroup of $G$. We assume toward a contradiction that $G$ has a Sylow $U_{K}$-subgroup $S$ non conjugate to $U$ and such that $S \cap C \neq 1$. We may assume:

for every Sylow $U_{K}$-subgroup $S_{0}$ of $G$, if $r k(S \cap C)<r k\left(S_{0} \cap C\right)$,

then $S_{0}$ and $U$ are conjugate. $(*)$

We show that $S \cap C$ is a homogeneous $U_{K}$-subgroup. Otherwise Fact 3.13 yields an interpretable field $K_{0} \neq K$ of characteristic 0 such that $U_{K_{0}}(S \cap C) \neq 1$. Consequently, since $U_{K_{0}}(S) \geq U_{K_{0}}(S \cap C)$ centralizes $S=U_{K}(S)$ (Fact 3.11), the subgroup $S$ is a Sylow $U_{K}$-subgroup of $N_{G}\left(U_{K_{0}}(S \cap C)\right)$, contradicting the choice of $S$ (Lemma 8.1 3.). Thus $S \cap C$ is a homogeneous $U_{K}$-subgroup, in particular we have $S \cap C=S \cap U$.

We consider $N_{S}=U_{K}\left(N_{S}(S \cap U)\right)$. By Fact 6.3 , we have $S \cap U<N_{S} \leq$ $N_{G}(S \cap C)$, and Lemma 8.1 3. gives $g \in G$ such that $N_{S}^{g} \leq U$. Then we have $r k(S \cap C)<r k\left(N_{S}\right)=r k\left(N_{S}^{g}\right) \leq r k\left(S^{g} \cap C\right)$ and $(*)$ provides the result.

Theorem 8.5. - Suppose that $G$ has a Carter subgroup D non conjugate to $C$ such that $C \cap D \neq 1$. Then $C$ is abelian and $U_{K}(C) \cap D=1$.

Proof - By Lemma 6.6, $U=U_{K}(C)$ is a Sylow $U_{K}$-subgroup of $G$. By Fact $3.13, U$ is a homogeneous $U_{K}$-subgroup. We show that $U \cap D=1$. We assume toward a contradiction that $U \cap D \neq 1$. Let $V=U_{K}(D)$. By Proposition 8.4, there exists $x \in G$ such that $V^{x} \leq U$. By Lemma 8.1 1. and 2., $N=N_{G}\left(V^{x}\right)^{\circ}$ is a solvable $\widetilde{V}$-subgroup and $r=\bar{r}_{\min }(N)$. By Lemma 4.9, and since $D^{x}$ normalizes $V^{x}$, $D^{x}$ contains a Sylow $U_{0, r}$-subgroup $R$ of $N$ and we obtain $V^{x}=U_{K}\left(D^{x}\right)=U_{K}(R)$. Consequently, by Fact $6.5, V^{x}$ is the unique Sylow $U_{K^{-}}$subgroup of $N$, in particular $U_{K}(N)=V^{x}$ and $V^{x}$ is a Sylow $U_{K}$-subgroup of $G$ (Fact 6.3). Hence $U=V^{x}$, and $C$ and $D$ are two non conjugate Carter subgroups of $N$, contradicting Fact 1.3. This proves that $U \cap D=1$ and, by Fact 6.8 , we obtain the result.

\section{The subgroups $V_{K}(\cdot)$ And $X_{K}(\cdot)$}

We introduce and we analyse the last ingredients for our proof. Subgroups $V_{K}(\cdot)$ and $X_{K}(\cdot)$ are to the heart of our proof and of our final rank calculation. The main result of this section is Theorem 9.10.

Notation 9.1. - If $H$ is a definable subgroup of $G$, we let:

- $V_{K}(H)=U_{K}\left(F\left(N_{G}(H)\right)\right)$;

- $X_{K}(H)=\left\langle U_{L}\left(N_{G}(H)\right)\right| L \neq K$ interpretable field of characteristic 0$\rangle$. 
Remark 9.2. - If $H$ is a definable subgroup of $G$, then $V_{K}(H)$ is a nilpotent

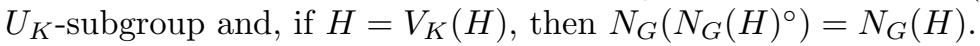

Moreover, if $H$ is a definable subgroup of $U_{K}(C)$, then $V_{K}(H)$ is a homogeneous $U_{K}$-subgroup (Proposition 8.4).

Lemma 9.3. - Let $H$ be a nontrivial definable subgroup of $U_{K}(C)$ and suppose $H=V_{K}(H)$. Then $U_{K}\left(X_{K}(H)\right)=1$, in particular $H \cap X_{K}(H)=1$ and $H$ centralizes $X_{K}(H)$.

Furthermore, if $D$ is a Carter subgroup of $N_{G}(H)$ and if $E=\left\langle U_{L}(D)\right| L \neq$ $K$ interpretable field of characteristic 0$\rangle$, then $H X_{K}(H)=F\left(N_{G}(H)\right)^{\circ} E$.

Proof - By Lemma 8.11 ., $N_{G}(H)^{\circ}$ is a solvable $\widetilde{V}$-group, in particular its definable nilpotent subgroups are torsion-free $\widetilde{U}$-subgroups (Corollary 3.17 ). By Lemma 8.12 . and by Fact 3.6, $D H$ is nilpotent, so $D$ contains $H$. We consider

$$
\left.U=\left\langle U_{L}\left(F\left(N_{G}(H)\right)\right)\right| L \neq K \text { interpretable field of characteristic } 0\right\rangle .
$$

Then Fact 3.13 gives $F\left(N_{G}(H)\right)^{\circ}=H \times U \leq D U$, and Facts 2.4 and 2.13 yield $N_{G}(H)^{\circ}=D U$. Now Fact 3.18 implies $U_{L}\left(N_{G}(H)\right) \leq U E$ for any interpretable field $L \neq K$ of characteristic zero, so $X_{K}(H)=U E$.

But Fact 3.13 gives $U_{K}(U)=U_{K}(E)=1$, therefore Fact 3.18 yields

$$
U_{K}(U E) U / U=U_{K}(U E / U)=U_{K}(E) U / U=1
$$

and $U_{K}\left(X_{K}(H)\right)=U_{K}(U E)=U_{K}(U)=1$. Since $H \leq U_{K}(C)$ is a homogeneous $U_{K}$-subgroup, we obtain $H \cap X_{K}(H)=1$ and $H$ centralizes $X_{K}(H)$. Furthermore the preceding paragraph and Fact 3.13 provide $H X_{K}(H)=H(U E)=(H U) E=$ $F\left(N_{G}(H)\right)^{\circ} E$.

Lemma 9.4. - Let $H$ be a nontrivial definable subgroup of $U_{K}(C)$ and suppose $H=V_{K}(H)$. Then $H$ is a Sylow $U_{K}$-subgroup of $C_{G}\left(X_{K}(H)\right)$.

Proof - By Lemma 9.3, we have $H \leq C_{G}\left(X_{K}(H)\right)$, so there is a Sylow $U_{K^{-}}$ subgroup $V$ of $C_{G}\left(X_{K}(H)\right)$ containing $H$. We consider $W=U_{K}\left(N_{V}(H)\right)$. Then $W$ is a nilpotent normal subgroup of $X_{K}(H) W$ and $W \leq F\left(X_{K}(H) W\right)$.

By Fact 2.4 and Lemma $8.11 ., F\left(N_{G}(H)^{\circ}\right)$ is a maximal nilpotent normal subgroup of $N_{G}(H)^{\circ}$. But Lemma 8.1 1. gives $\widetilde{U}\left(N_{G}(H)^{\circ}\right)=F\left(N_{G}(H)^{\circ}\right)$ and Fact 3.13 yields $\widetilde{U}\left(N_{G}(H)^{\circ}\right) \leq X_{K}(H) H \leq X_{K}(H) W$, hence $F\left(N_{G}(H)^{\circ}\right)=$ $F\left(X_{K}(H) W\right)$ and we obtain $W \leq F\left(N_{G}(H)^{\circ}\right) \leq F\left(N_{G}(H)\right)$. This proves that $W$ is contained in $V_{K}(H)=H$, and Fact 6.3 says that $H=W$ is a Sylow $U_{K^{-}}$ subgroup of $V$, so $H=V$.

The following proposition is our first important result concerning the subgroups of Notation 9.1 .

Proposition 9.5. - Let $H$ be a definable subgroup of $U_{K}(C)$ and suppose $H=$ $V_{K}(H)$. Then, for any definable subgroup $M$ of $H$, we have $V_{K}(M) \leq H$.

Proof - We assume toward a contradiction that $H$ is a counterexample of minimal Morley rank and that $V_{K}(M) \not \leq H$. We may assume $V_{K}\left(M_{0}\right) \leq H$ for each definable subgroup $M_{0}$ of $H$ such that $M<M_{0}$. If $M=1$, then $F\left(N_{G}(M)\right)=1$ since $G$ is a $C^{*}$-group, so $V_{K}(M)=1$. This contradiction gives $M \neq 1$ and, by Proposition 8.4, $V_{K}(M)$ is a nilpotent homogeneous $U_{K}$-subgroup. 
We show that $X_{K}(H)$ centralizes $V_{K}(M)$. By Lemma 9.4, $H$ is a Sylow $U_{K^{-}}$ subgroup of $C_{G}\left(X_{K}(H)\right)$, in particular $X_{K}(H) \leq N_{G}(M)$. On the other hand, by Lemma 8.11 . and 2., $N_{G}(M)^{\circ}$ is a solvable $\widetilde{\widetilde{V}}$-group and $r=\bar{r}_{\min }\left(N_{G}(M)\right)$. Hence, for each nontrivial indecomposable $U_{L}$-subgroup $A$ of $N_{G}(M)$ with $L \neq K$ an interpretable field of characteristic zero, we have $r k(L) \geq r$ and Fact 3.6 says that $A V_{K}(M)$ is nilpotent, so $A$ centralizes $V_{K}(M)$ (Fact 3.10). This proves that $X_{K}(H)$ centralizes $V_{K}(M)$.

Let $P=N_{H}(M)>M$. Then $P$ normalizes $V_{K}(M)$ and $P V_{K}(M)$ is nilpotent (Fact 3.6). Since $V_{K}(M) \not H$, we have $P<P V_{K}(M)$ and $N_{V_{K}(M)}(P) \not \leq P$. Since $N_{V_{K}(M)}(P) \leq V_{K}(M)$ normalizes $M$, we obtain $N_{V_{K}(M)}(P) \not \leq H$.

By the preceding paragraph, there exists a definable subgroup $N$ of $H$, such that $M<N$ and $N_{V_{K}(M)}(N) \not \leq H$. We consider such a subgroup $N$ of maximal rank. Then the choice of $M$ implies $V_{K}(N) \leq H$. Let $U_{0}=N$ and, for each $i \in \mathbb{N}$ such that $U_{i} \leq H, U_{i+1}=V_{K}\left(U_{i}\right)$. By Proposition 8.4, $U_{i}$ is a homogeneous $U_{K}$-subgroup for each $i$, so this sequence is increasing and our hypothesis over $H$ and $M$ implies $U_{i} \leq H$ for each $i$. In particular there exists $j \in \mathbb{N}$ such that $U_{j}=U_{j+1}=V_{K}\left(U_{j}\right)$, and we have $U_{j}=H$ by the minimality of $r k(H)$. Since $X_{K}(N)$ normalizes $N=U_{0}$, we have $X_{K}(N) \leq N_{G}\left(U_{j}\right)=N_{G}(H)$, so $X_{K}(N) \leq X_{K}(H)$. Moreover, since $N \leq H$ centralizes $X_{K}(H)$ (Lemma 9.4), we have $X_{K}(H) \leq N_{G}(N)$ and $X_{K}(H)=X_{K}(N)$. Consequently, by the second paragraph, $V_{K}(M)$ centralizes $X_{K}(N)$ and, if $N_{V}=N_{V_{K}(M)}(N)$, then $E_{N_{G}(N)^{\circ}}\left(N_{V}\right)$ contains $X_{K}(N)$. But $N_{G}(N)^{\circ}$ is a solvable $\widetilde{V}$-group (Lemma 8.11 .), so Fact 3.18 shows that $N_{G}(N)^{\circ} / X_{K}(N)$ is a solvable homogeneous $U_{K^{-}}$group, and it is nilpotent by Fact 3.12. Now Fact 2.15 gives $N_{G}(N)^{\circ}=E_{N_{G}(N)^{\circ}}\left(N_{V}\right) X_{K}(N)$, hence $E_{N_{G}(N)^{\circ}}\left(N_{V}\right)=N_{G}(N)^{\circ}$, and we have $N_{V} \leq F\left(N_{G}(N)^{\circ}\right) \leq F\left(N_{G}(N)\right.$ ) (Fact 2.14). Since $V_{K}(M)$ is a homogeneous $U_{K}$-subgroup, we obtain $N_{V} \leq V_{K}(N) \leq H$, contradicting $N_{V} \nless \leq H$.

Corollary 9.6. - Let $D$ be a conjugate of $C$. Let $H$ (resp. L) be a definable subgroup of $U_{K}(C)$ (resp. $U_{K}(D)$ ) such that $H=V_{K}(H)$ (resp. $\left.L=V_{K}(L)\right)$. Then we have $H \cap L=V_{K}(H \cap L)$.

Proof - Since $H=V_{K}(H) \leq U_{K}(C)$ is a homogeneous $U_{K}$-subgroup, then $H \cap L$ is a $U_{K}$-subgroup too and $H \cap L$ is contained in $V_{K}(H \cap L)$. Moreover, by Proposition 9.5, we have $V_{K}(H \cap L) \leq H \cap L$.

Fact 9.7. - [16, Remark B.2 (iii)] If $H$ is a group of finite Morley rank, there is a bound on the Morley degrees of any uniformly definable family of subgroups of $H$.

Fact 9.8. - [20, Theorem 1.4.3] Let L a subgroup of a group $H$ of finite Morley rank. Then $F(L)=\left\{x \in L \mid \exists n \in \mathbb{N}, \forall y \in L,\left[y,_{n} x\right]=1\right\}$.

Lemma 9.9. - If $\mathscr{F}=\left\{L_{i} \mid i \in I\right\}$ is a uniformly definable family of subgroups of a group $H$ of finite Morley rank, then $\left\{F\left(L_{i}\right) \mid i \in I\right\}$ is a uniformly definable family of subgroups of $H$.

Proof - By Fact 9.7, there exists an integer $d$ such that $\operatorname{deg}\left(L_{i}\right) \leq d$ for each $i \in I$. Let $n=r k(H)+d+1$. We have only to prove that, for every $L \in \mathscr{F}, F(L)=\left\{x \in L \mid \forall y \in L,\left[y,_{n} x\right]=1\right\}$. Let $L \in \mathscr{F}$ and $x \in F(L)$. We have $[L, x] \leq F(L)$ and, since $F(L) L^{\circ} / L^{\circ} \leq L / L^{\circ}$ has a nilpotence class at most $d-1,\left[y,_{d} x\right] \in F(L) \cap L^{\circ}$ for all $y \in L$. But $\left[L^{\circ}, x\right] \leq F(L)^{\circ}$ and we have 
$F(L)^{\circ} \leq Z_{r k(L)}(F(L)) \leq Z_{r k(H)}(F(L))$, so $\left[y,_{n} x\right]=1$ for all $y \in L$. This proves that $F(L)$ is contained in $\left\{x \in L \mid \forall y \in L,\left[y,_{n} x\right]=1\right\}$. Now Fact 9.8 finishes the proof.

Theorem 9.10. - Let $\mathscr{F}=\left\{F_{i} \mid i \in I\right\}$ be a uniformly definable family of subgroups of $U_{K}(C)$ such that $F=V_{K}(F)$ for each $F \in \mathscr{F}$. Then $\mathscr{E}=\left\{F_{i} X_{K}\left(F_{i}\right) \mid i \in I\right\}$ is a uniformly definable family of subgroups of $G$.

Proof - We may assume $F \neq 1$ for each $F \in \mathscr{F}$. Since $\left\{N_{G}(F) \mid F \in \mathscr{F}\right\}$ is a uniformly definable family of subgroups of $G$, Fact 9.7 says that there is a bound $b$ on the Morley degrees of $N_{G}(F)$ for $F \in \mathscr{F}$. If $c=b$ !, then the order of $N_{G}(F) / N_{G}(F)^{\circ}$ divides $c$ for each $F \in \mathscr{F}$. By Lemma 8.1 1., $N_{G}(F)^{\circ}$ is torsion-free for each $F \in \mathscr{F}$, in particular $N_{G}(F)^{\circ}$ is divisible and $N_{G}(F)^{\circ}=\left\{g^{c} \mid g \in N_{G}(F)\right\}$. This proves that $\left\{N_{G}\left(F_{i}\right)^{\circ} \mid i \in I\right\}$ is a uniformly definable family of subgroups of $G$. Now Lemma 9.9 proves that $\left\{F\left(N_{G}\left(F_{i}\right)^{\circ}\right) \mid i \in I\right\}$ is a uniformly definable family of subgroups of $G$. But, by Lemma 8.1 1. and by Fact 3.17, $F\left(N_{G}\left(F_{i}\right)\right)^{\circ}$ is a $\widetilde{U}$-subgroup for each $i \in I$, hence Fact 3.13 yields $F\left(N_{G}\left(F_{i}\right)\right)^{\circ} \leq F_{i} X_{K}\left(F_{i}\right)$ for each $i \in I$. Moreover, since $N_{G}\left(F_{i}\right)^{\circ}$ is torsion-free, we have $F\left(N_{G}\left(F_{i}\right)^{\circ}\right)=F\left(N_{G}\left(F_{i}\right)\right)^{\circ}$ for each $i \in I$, and $\left\{F\left(N_{G}\left(F_{i}\right)\right)^{\circ} \mid i \in I\right\}$ is a uniformly definable family of subgroups of $G$.

Case 1: $C$ is abelian.

Let $E=\left\langle U_{K_{0}}(C)\right| K_{0} \neq K$ interpretable field of characteristic 0$\rangle$. For each $F \in \mathscr{F}$, we have $C \leq C_{G}(F) \leq N_{G}(F)$ and $C$ is a Carter subgroup of $N_{G}(F)$. Consequently, by Lemma 9.3 , we have $F X_{K}(F)=F\left(N_{G}(F)\right)^{\circ} E$ for each $F \in \mathscr{F}$. This proves that $\mathscr{E}=\left\{F\left(N_{G}\left(F_{i}\right)\right)^{\circ} E \mid i \in I\right\}$ is a uniformly definable family of subgroups of $G$.

Case 2: $C$ is non abelian.

By Fact $6.8, C$ is a homogeneous $U_{K}$-subgroup. By Corollary 8.3, $C$ is contained in a unique maximal $C$-subgroup $B$ of $G$, and $B$ is a solvable $\widetilde{V}$-group. Let $H=$ $\left\langle U_{K_{0}}(F(B))\right| K_{0} \neq K$ interpretable field of characteristic 0$\rangle$. By Facts 3.13 and 3.17, we have $F(B)=H \times U_{K}(F(B))$, in particular $U_{K}(H)=1$ and

$$
N_{H}(F) \leq X_{K}(F) \text { for each } F \in \mathscr{F}(1) \text {. }
$$

By Facts 2.4 and 2.13, we have $B=F(B) C$, and Fact 3.6 gives $U_{K}(F(B)) \leq C$, so $B=H C$. Since $U_{K}(H)=1$ and since $C$ is a homogeneous $U_{K}$-subgroup, we obtain $B=H \rtimes C$.

We show that $N_{C}\left(N_{C}(F)\right)$ is nonabelian for each $F \in \mathscr{F}$. Indeed, we may assume that $N_{C}(F)$ is abelian for $F \in \mathscr{F}$. Then $F$ is abelian and $N_{C}(F)$ is a maximal abelian subgroup of $C$ containing $F$. Since $C$ is non abelian, we obtain $N_{C}(F)<C$ and $N_{C}(F)<N_{C}\left(N_{C}(F)\right)$, so $N_{C}\left(N_{C}(F)\right)$ is non abelian.

We show that $N_{G}\left(N_{C}(F)\right)^{\circ}$ is contained in $B$ for each $F \in \mathscr{F}$. Let $F \in \mathscr{F}$. By the preceding paragraph, $N_{C}\left(N_{C}(F)\right)$ is nonabelian and, by Corollary $8.3, B$ is the unique maximal $C$-subgroup of $G$ containing $N_{C}\left(N_{C}(F)\right)$. But $N_{G}\left(N_{C}(F)\right)^{\circ}$ is a $C$-group since there is a $N_{G}\left(N_{C}(F)\right)^{\circ}$-minimal subgroup $A$ in $N_{C}(F)$, and $A$ is abelian and divisible as $C$ is a nilpotent and torsion-free, so $N_{G}\left(N_{C}(F)\right)^{\circ}$ is contained in $B$.

Let $F \in \mathscr{F}$. We show that $F X_{K}(F)=F\left(N_{G}(F)\right)^{\circ} N_{H}(F)$. By $(*)$, this is sufficient to conclude. Since $N_{G}(F)^{\circ}$ is a solvable $\widetilde{V}$-group (Lemma 8.11 .), 
Fact 2.14 shows that $D=E_{X_{K}(F) N_{C}(F)}\left(N_{C}(F)\right)$ is a connected definable subgroup of $N_{G}(F)^{\circ}$ and that $N_{C}(F)$ is contained in $F(D)$, so $N_{C}(F) \leq U_{K}(F(D)$ ). Since $U_{K}(F(D)) \cap C$ contains $F \neq 1$, Proposition 8.4 says that $U_{K}(F(D))$ is a homogeneous $U_{K}$-subgroup, in particular $U_{K}(F(D)) \cap X_{K}(F)$ is contained in $U_{K}\left(X_{K}(F)\right)=1$ (Lemma 9.3). Thus we have

$$
U_{K}(F(D))=\left(U_{K}(F(D)) \cap X_{K}(F)\right) N_{C}(F)=N_{C}(F),
$$

in particular $D$ normalizes $N_{C}(F)$, and $D$ is contained in $B$ by the preceding paragraph. Since $U_{K}\left(X_{K}(F)\right)=1$ (Lemma 9.3) and since $B=H \rtimes C$ with $C$ a homogeneous $U_{K}$-subgroup, Fact 3.18 gives

$$
\left(B \cap X_{K}(F)\right) H / H=U_{K}\left(\left(B \cap X_{K}(F)\right) H / H\right)=U_{K}\left(B \cap X_{K}(F)\right) H / H=1,
$$

so $B \cap X_{K}(F)$ is contained in $H$, in particular $D \cap X_{K}(F) \leq H$. Thus we obtain

$$
F X_{K}(F) \cap D=\left(X_{K}(F) \cap D\right) F \leq(H \cap D) F \leq N_{H}\left(N_{C}(F)\right) F .
$$

Since $\left[N_{H}\left(N_{C}(F)\right), F\right] \leq H \cap N_{C}(F)=1$, we have

$$
F X_{K}(F) \cap D \leq N_{H}(F) F(2) \text {. }
$$

Also, since $N_{G}(F)^{\circ}$ is a solvable $\widetilde{V}$-group, $F\left(N_{G}(F)\right)^{\circ}$ is a $\widetilde{U}$-subgroup and Fact 3.13 yields

$$
F\left(N_{G}(F)\right)^{\circ} \leq V_{K}(F) X_{K}(F)=F X_{K}(F)(3) .
$$

By Fact 2.15, we have $X_{K}(F) N_{C}(F)=\left(X_{K}(F) N_{C}(F)\right)^{\prime} D$, and Facts 2.4 and 2.13 imply

$$
X_{K}(F) N_{C}(F)=\left(X_{K}(F) N_{C}(F)\right)^{\prime} D \leq F\left(N_{G}(F)\right)^{\circ} D(4) .
$$

Now, since $F=V_{K}(F)$ is contained in $F\left(N_{G}(F)\right)^{\circ}(5)$, we obtain

$$
\begin{aligned}
F X_{K}(F) & =F\left(N_{G}(F)\right)^{\circ} D \cap F X_{K}(F) & & \text { by (4) } \\
& =F\left(N_{G}(F)\right)^{\circ}\left(D \cap F X_{K}(F)\right) & & \text { by (3) } \\
& \leq F\left(N_{G}(F)\right)^{\circ}\left(N_{H}(F) F\right) & & \text { by (2) } \\
& =F\left(N_{G}(F)\right)^{\circ} N_{H}(F) & & \text { by (5) } \\
& \leq F\left(N_{G}(F)\right)^{\circ} X_{K}(F) & & \text { by (1) } \\
& \leq F X_{K}(F) & & \text { by (3) }
\end{aligned}
$$

This proves that $F X_{K}(F)=F\left(N_{G}(F)\right)^{\circ} N_{H}(F)$ and finishes our proof.

\section{A Generosity theorem}

In this section, we describe the structure of $G$ (Theorem 10.6). The main ingredient for the proof is Theorem 10.5.

Lemma 10.1. - Let $B$ be a maximal $C$-subgroup of $G$ containing $C$. If $C=$ $U_{K}(C)$, then $B=H \rtimes C$ for a definably characteristic nilpotent $\widetilde{V}$-subgroup $H$ of B. Moreover, $C$ is a Sylow $U_{0, r}$-subgroup of $G$ and $U_{0, r}(H)=1$.

Proof - By Proposition 8.2, $B$ is a solvable $\widetilde{V}$-subgroup, in particular $F(B)$ is a torsion-free $\widetilde{U}$-subgroup (Corollary 3.17 ). Then Fact 3.13 yields $F(B)=H \times$ $U_{K}(F(B))$ where

$$
\left.H=\left\langle U_{L}(F(B))\right| L \neq K \text { is an interpretable field of characteristic } 0\right\rangle,
$$

so $H$ is a definably characteristic nilpotent $\widetilde{V}$-subgroup of $B$. 
But Fact 3.6 provides the nilpotence of $U_{0, r}(F(B)) C$, hence we have $C \geq$ $U_{0, r}(F(B)) \geq U_{K}(F(B))$. Now, as Facts 2.4 and 2.13 give $B=F(B) C$, we obtain $B=H C$ and, as $C=U_{K}(C)$ is a homogeneous $U_{K}$-subgroup, we have

$$
H \cap C \leq H \cap(F(B) \cap C) \leq H \cap U_{K}(F(B))=1 .
$$

Moreover, since $C$ is a $U_{K}$-subgroup, it is a $U_{0, r}$-subgroup and $U_{0, r}\left(N_{G}(C)\right)=$ $U_{0, r}(C)=C$, consequently Fact 3.3 shows that $C$ is a Sylow $U_{0, r}$-subgroup of $G$. Finally, we have $U_{0, r}(H) \leq H \cap U_{0, r}(F(B)) \leq H \cap C=1$.

Proposition 10.2 corresponds to the well-known notion of Control of Fusion [3, Section 10.6.1 p.187].

Proposition 10.2. - If $A$ is a definable subgroup of $C$, then for every $g \in G$ such that $A^{g} \leq C$, there exists $x \in N_{G}(C)$ such that $A^{x}=A^{g}$.

Proof - We may assume $A \neq 1$. Let $g \in G$ suh that $A^{g} \leq C$. If $C$ is abelian, then $C$ and $C^{g^{-1}}$ are two Carter subgroups of the $C$-group $C_{G}(A)^{\circ}$, and there exists $y \in C_{G}(A)$ such that $C^{y}=C^{g^{-1}}$. In particular we have $y g \in N_{G}(C)$ and $A^{y g}=A^{g}$, proving the existence of $x=y g \in N_{G}(C)$ such that $A^{x}=A^{g}$. Hence we may assume $C$ non abelian.

By Fact $6.8, C$ is a homogeneous $U_{K}$-subgroup. By Corollary 8.3 , there is a unique maximal $C$-subgroup $B$ of $G$ containing $C$, and $B$ is a solvable $\widetilde{V}$-subgroup. Consequently, by Lemma 10.1, we have $B=H \rtimes C$ for $H$ a definably characteristic nilpotent $\widetilde{V}$-subgroup of $B$ such that $U_{0, r}(H)=1$.

We show that,

for every $u \in N_{G}(B)$ such that $A^{u} \leq C$, there exists $x \in N_{G}(C)$ such that $A^{x}=A^{u}(*)$.

Indeed, for every $u \in N_{G}(B)$ such that $A^{u} \leq C$, there exists $b$ an element of the $C$-subgroup $B$ such that $C^{u b}=C$, and we have $A^{u b} \leq C$. Since $B=H \rtimes C$, we may assume $b \in H$, in particular we obtain $H \rtimes A^{u}=H \rtimes A^{u b}$ and $A^{u}=$ $C \cap\left(H \rtimes A^{u}\right)=C \cap\left(H \rtimes A^{u b}\right)=A^{u b}$. Since $u b \in N_{G}(C)$, this proves $(*)$.

By $(*)$ we may assume $g \notin N_{G}(B)$. In particular we have $B \neq B^{g^{-1}}$ and, since $A^{g} \leq C \leq B$, we have $A \leq B^{g^{-1}} \cap B$ and $A$ is abelian (Corollary 8.3).

Let $U=N_{C}\left(A^{g}\right)$ and $b \in N_{B}\left(A^{g}\right)$. Then there exist $h \in H$ and $c \in C$ such that $b=h c$ and, for each $a \in A$, we have $\left[h, a^{g}\right]^{c}\left[c, a^{g}\right]=\left[h c, a^{g}\right]=\left[b, a^{g}\right] \in A^{g} \leq C$. Since $\left[h, a^{g}\right]^{c} \in H$ and $\left[c, a^{g}\right] \in C$, we obtain $\left[h, a^{g}\right]^{c} \in H \cap C=1$ and $h \in C_{H}\left(a^{g}\right)$. Thus $c=h^{-1} b$ belongs to $N_{C}\left(A^{g}\right)=U$, and we obtain $N_{B}\left(A^{g}\right)=C_{H}\left(A^{g}\right) \rtimes U$. In the same way, if we let $V=N_{C}(A)^{g}$, then $N_{B}(A)^{g}=C_{H}(A)^{g} \rtimes V$.

We show that $U$ and $V$ are abelian. We suppose toward a contradiction that $U$ is non abelian. Then $B$ is the unique maximal $C$-subgroup of $G$ containing $U$ (Corollary 8.3). Moreover $A$ is abelian and divisible, so $N_{G}(A)^{\circ}$ is a $C$-group. Since $U$ is contained in $N_{B}\left(A^{g}\right) \leq N_{G}\left(A^{g}\right)^{\circ}=\left(N_{G}(A)^{\circ}\right)^{g}$, we obtain $\left(N_{G}(A)^{\circ}\right)^{g} \leq B$, and $V \leq B \cap B^{g}$. Henceforth $V$ is abelian (Corollary 8.3) and $V$ is the unique maximal abelian subgroup of $C^{g}$ containing $A^{g}$. Since $C^{g}$ is non abelian, we have $V<C^{g}$, therefore $V<N_{C^{g}}(V)$ and $N_{C^{g}}(V)$ is non abelian. In particular $B^{g}$ is the unique maximal $C$-subgroup of $G$ containing $N_{G}(V)^{\circ}$ (Corollary 8.3). Let $W$ be a Sylow $U_{0, r}$-subgroup of $B$ containing $V$. By Fact 3.4 and Lemma 10.1, $C=U_{K}(C)$ 
is conjugate to $W$. In particular $W$ is non abelian and, since $V$ is abelian, we have $V<W$ and $V<N_{W}(V)$. But we have

$$
N_{W}(V) \leq N_{G}(V)^{\circ} \cap B \leq B^{g} \cap B,
$$

so $N_{W}(V)$ is abelian (Corollary 8.3), and we obtain

$$
N_{W}(V) \leq B^{g} \cap N_{G}\left(A^{g}\right)=N_{B}(A)^{g}=C_{H}(A)^{g} \rtimes V .
$$

Since $V<N_{W}(V)$, this contradicts $N_{W}(V) \cap C_{H}(A)^{g} \leq W \cap H^{g} \leq U_{K}(H)^{g}=1$. Hence $U$ is abelian. In the same way, $V$ is abelian.

Since $U$ is abelian, $U$ is the unique maximal abelian subgroup of $C$ containing $A^{g}$. Since $C$ is non abelian, we have $U<C$ and $U<N_{C}(U)$, therefore $N_{C}(U)$ is non abelian. In particular, by Corollary 8.3, $B$ is the unique maximal $C$-subgroup of $G$ containing $N_{C}(U)$. Since $U$ is abelian and divisible, $N_{G}(U)^{\circ}$ is a $C$-group, so $B$ is the unique maximal $C$-subgroup of $G$ containing $N_{G}(U)^{\circ}$. In the same way, $B^{g}$ is the unique maximal $C$-subgroup of $G$ containing $N_{G}(V)^{\circ}$.

We show that $U$ and $V$ are conjugate in $\left(N_{G}(A)^{g}\right)^{\circ}$. Let $U_{1}$ be a Sylow $U_{0, r^{-}}$ subgroup of $N_{G}(A)^{g}$ containing $U$. Then $U_{2}=U_{0, r}\left(N_{U_{1}}(U)\right)$ is contained in $N_{G}(U)^{\circ} \leq B$. Now Lemma 10.1 and Fact 3.4 say that $U_{2}$ is $B$-conjugate with a subgroup of $C$, so $U_{2} \cap H=1$. But $U_{2}$ contains $U$ and is contained in $N_{B}\left(A^{g}\right)=$ $C_{H}\left(A^{g}\right) \rtimes U$, hence $U_{2}=U$. Then Fact 3.3 give $U=U_{1}$ and $U$ is a Sylow $U_{0, r^{-}}$ subgroup of $N_{G}(A)^{g}$. In the same way, $V$ is a Sylow $U_{0, r}$-subgroup of $N_{G}(A)^{g}$. Since $N_{G}(A)^{g}$ is a $C$-subgroup of $G$, it is solvable (Proposition 8.2), and Fact 3.4 yields $y \in\left(N_{G}(A)^{g}\right)^{\circ}$ such that $V^{y}=U$.

Consequently we obtain $N_{G}(U)^{\circ}=\left(N_{G}(V)^{\circ}\right)^{y} \leq B^{g y} \cap B$ and, since $B$ is the unique maximal $C$-subgroup of $G$ containing $N_{G}(U)^{\circ}$, we find $B=B^{g y}$. Moreover we have $y \in\left(N_{G}(A)^{g}\right)^{\circ}=N_{G}\left(A^{g}\right)^{\circ}$, so $A^{g y}=A^{g} \leq C$. Now we apply $(*)$ with $u=g y$, and we obtain $x \in N_{G}(C)$ such that $A^{x}=A^{g}$.

Proposition 10.3. - Let $R$ be a solvable $\widetilde{V}$-group, and $U$ a Sylow $U_{K}$-subgroup of $R$. Then $\left\{U \cap U^{g} \mid g \in R\right\}$ is a finite set.

Proof - We proceed by induction on $r k(R)$. By Fact 6.5 , we may assume $R=R^{\prime} U$. By Corollary 3.17 and by Fact $3.13, U$ is a homogeneous $U_{K}$-subgroup.

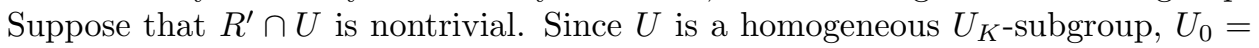
$U_{K}\left(R^{\prime}\right)$ is nontrivial too. By Fact 2.4, $R^{\prime}$ is nilpotent, therefore Fact 3.6 gives $U_{0} \leq U$. Then Fact 3.18 yields $U_{K}\left(N_{R}(U)\right)=U_{K}\left(N_{R^{\prime}}(U) U\right)=U_{K}\left(N_{R^{\prime}}(U)\right) U=U$ and

$$
\left.U_{K}\left(N_{R / U_{0}}\left(U / U_{0}\right)\right)=U_{K}\left(N_{R}(U) / U_{0}\right)\right)=U_{K}\left(N_{R}(U)\right) / U_{0}=U / U_{0}
$$

so $U / U_{0}$ is a Sylow $U_{K}$-subgroup of $R / U_{0}$ (Fact 6.3). Moreover, $R / U_{0}$ is a $\widetilde{V}$ group by Facts 3.1 and 3.16. Hence, by the induction hypothesis, we may assume $R=R^{\prime} \rtimes U$. Note that the equality $R=R^{\prime} \rtimes U$ implies, in particular, that $U$ is abelian and that $N_{R}(U)$ centralizes $U$.

We show that, for every $g \in R$ we have $U \cap U^{g}=U_{K}\left(F\left(E_{R}\left(U \cap U^{g}\right)\right)\right)$. By Fact 2.14, for every $g \in R$, we have $U \cap U^{g} \leq U_{K}\left(F\left(E_{R}\left(U \cap U^{g}\right)\right)\right)$. Also Fact 3.6 says that, for every $g \in R, U^{g} U_{K}\left(F\left(E_{R}\left(U \cap U^{g}\right)\right)\right)$ is a nilpotent $U_{K}$-subgroup, so $U_{K}\left(F\left(E_{R}\left(U \cap U^{g}\right)\right)\right) \leq U^{g}$. Hence we obtain $U \cap U^{g}=U_{K}\left(F\left(E_{R}\left(U \cap U^{g}\right)\right)\right)$ for every $g \in R$.

We show that, if there exists $x \in R$ such that $\left(U \cap U^{g}\right)^{x}=U \cap U^{h}$ for $g$ and $h$ two elements of $R$, then $U \cap U^{g}=U \cap U^{h}$. Indeed, we have $U \cap U^{g} \leq U \cap U^{x^{-1}}$ 
and, by Fact 6.5 , there exists $y \in C_{R}\left(U \cap U^{g}\right)$ such that $U^{y}=U^{x^{-1}}$. In particular we have $U^{y x}=U$ and $y x \in N_{R}(U)=C_{R}(U)$, so

$$
U \cap U^{g}=\left(U \cap U^{g}\right)^{y x}=\left(U \cap U^{g}\right)^{x}=U \cap U^{h} .
$$

Let $g$ and $h$ be two elements of $R$ such that $U \cap U^{g} \neq U \cap U^{h}$. By the preceding paragraph, $U \cap U^{g}$ and $U \cap U^{h}$ are not conjugate and, since $U \cap U^{\alpha}=U_{K}\left(F\left(E_{R}(U \cap\right.\right.$ $\left.\left.U^{\alpha}\right)\right)$ ) for $\alpha \in\{g, h\}$, the subgroups $E_{R}\left(U \cap U^{g}\right)$ and $E_{R}\left(U \cap U^{h}\right)$ are not conjugate. Now Fact 2.16 yields the result.

Lemma 10.4. - Let $F$ be a nontrivial definable subgroup of $U_{K}(C)$ and suppose $F=V_{K}(F)$. Then $N_{G}(F)^{\circ}=X_{K}(F) \rtimes N_{U_{K}(C)}(F)$. Moreover, either $N_{U_{K}(C)}(F)$ is abelian and $N_{G}(F)^{\circ}$ centralizes $F$, or $N_{U_{K}(C)}(F)$ is nonabelian and $N_{U_{K}(C)}(F)=$ $C$.

Proof - Let $U=U_{K}(C)$. By Fact 3.13, it is a homogeneous $U_{K}$-subgroup. As $F$ is nilpotent, $F$ contains a $N_{G}(F)^{\circ}$-minimal subgroup, which is abelian (Fact 2.5), so $N_{G}(F)^{\circ}$ is a $C$-subgroup. By Proposition 8.2, $N_{G}(F)^{\circ}$ is a solvable $\widetilde{V}$-subgroup and $\bar{r}_{\text {min }}\left(N_{G}(F)^{\circ}\right)=r$, so its nilpotent definable subgroups are $\widetilde{U}$-subgroups (Corollary 3.17 ), in particular its $U_{K}$-subgroups are homogeneous (Fact 3.13).

Since $N_{U}(F)$ is a $U_{K}$-subgroup, Fact 3.4 and Lemma 4.9 provide a Carter subgroup $D$ of $N_{G}(F)$ containing $N_{U}(F)$. Since $D$ is a $\widetilde{U}$-subgroup, we have $D=E \times U_{K}(D)$ where $E=\left\langle U_{L}(D)\right| L \neq K$ interpretable field of characteristic 0$\rangle$ (Fact 3.13), and Facts 2.4 and 2.13 yield $N_{G}(F)^{\circ}=\left(F\left(N_{G}(F)\right)^{\circ} E\right) U_{K}(D)$. Now Lemma 9.3 gives $F\left(N_{G}(F)\right)^{\circ} E=X_{K}(F) F$ and $U_{K}\left(X_{K}(F)\right)=1$, so we have $U_{K}(D) \cap X_{K}(F)=1$ and

$$
N_{G}(F)^{\circ}=\left(X_{K}(F) F\right) U_{K}(D)=X_{K}(F) \rtimes U_{K}(D) .
$$

By Proposition 8.4, there exists $g \in G$ such that $U$ contains $U_{K}(D)^{g}$, in particular $C$ contains $F^{g}$. Now Proposition 10.2 provides $x \in N_{G}(C)$ such that $F^{x}=F^{g}$, that is $g x^{-1} \in N_{G}(F)$. Consequently, we obtain $U_{K}(D)^{g x^{-1}} \leq N_{G}(F)^{g x^{-1}}=N_{G}(F)$. Moreover, since $U$ contains $U_{K}(D)^{g}$ and since $x \in N_{G}(C) \leq N_{G}(U)$, we have $U_{K}(D)^{g x^{-1}} \leq U^{x^{-1}}=U$ and $U_{K}(D)^{g x^{-1}} \leq N_{U}(F)$. This implies $r k\left(U_{K}(D)\right) \leq$ $r k\left(N_{U}(F)\right)$. But $D$ contains the $U_{K}$-subgroup $N_{U}(F)$, hence $N_{U}(F) \leq U_{K}(D)$, and the equality $U_{K}(D)=N_{U}(F)$ holds. This proves that $N_{G}(F)^{\circ}=X_{K}(F) \rtimes N_{U}(F)$.

Suppose that $N_{U}(F)$ is abelian. Then $F$ centralizes $N_{U}(F)$ and, as lemma 9.3 says that $F$ centralizes $X_{K}(F)$, the subgroup $N_{G}(F)^{\circ}$ centralizes $F$.

Suppose that $N_{U}(F)$ is nonabelian. Then Corollary 8.3 yields a unique maximal $C$-subgroup $B$ containing $N_{U}(F)$, in particular $B$ contains $N_{G}(F)^{\circ}$ and $C$. Furthermore Corollary 8.3 says that $B$ is solvable. By Fact $2.14, E_{B}(F)$ is a connected definable subgroup of $B$ containing $N_{G}(F)^{\circ}$ and $C$, and we have $F \leq F\left(E_{B}(F)\right)$. Since $F$ is a $U_{K}$-subgroup, we obtain $F \leq U_{K}\left(F\left(E_{B}(F)\right)\right)$. We consider the nilpotent $U_{K}$-subgroup $S=U_{K}\left(N_{F\left(E_{B}(F)\right)}(F)\right)$, which contains $F$. Then $S$ is contained in $N_{G}(F)^{\circ}$, and it is normalized by $N_{G}(F)^{\circ} \leq E_{B}(F)$. Thus we have

$$
S \leq U_{K}\left(F\left(N_{G}(F)^{\circ}\right)\right)=U_{K}\left(F\left(N_{G}(F)\right)^{\circ}\right)=V_{K}(F)=F,
$$

and Fact 6.3 shows that $F$ is a Sylow $U_{K}$-subgroup of $F\left(E_{B}(F)\right)$, that is $F=$ $U_{K}\left(F\left(E_{B}(F)\right)\right)$. Hence $F$ is normalized by $E_{B}(F)$, in particular $C$ normalizes $F$ and $N_{U}(F)=U$. But $C \geq N_{U}(F)$ is nonabelian, so Fact 6.8 provides $C=U$, and this equality finishes our proof. 
Theorem 10.5. - Let $H$ be a proper definable subgroup of $U_{K}(C)$ and suppose $H=V_{K}(H)$. Let $\mathscr{F}=\left\{F_{i} \mid i \in I\right\}$ be a uniformly definable family of proper subgroups of $H$, with $I$ a definable set, and such that $F_{i}=V_{K}\left(F_{i}\right)$ for each $i \in I$. If $\cup \mathscr{F}$ is generic in $H$, then $C$ is a nonabelian $U_{K}$-subgroup, and there are a solvable connected definable subgroup $B$ containing $C$ and $g \in B \backslash N_{B}(C)$ such that $H \leq C^{g}$.

Proof - We may assume $F_{i} \neq 1$ for each $i \in I$, in particular $N_{G}\left(F_{i}\right)^{\circ}$ is a $C$-subgroup. For each $i \in I$, we consider $B_{i}$ a maximal $C$-subgroup of $G$ containing $N_{G}\left(F_{i}\right)^{\circ}$. By Proposition 8.2, it is a solvable $\widetilde{V}$-subgroup, in particular its $U_{K^{-}}$ subgroups are homogeneous (Fact 3.13 and Corollary 3.17).

Suppose that $\mathscr{B}=\left\{B_{i} \mid i \in I\right\}$ is a finite set. Then there exists $i_{0} \in I$ such that, if we let $\mathscr{F}_{0}=\left\{F \in \mathscr{F} \mid N_{G}(F)^{\circ} \leq B_{i_{0}}\right\}$, the union $\cup \mathscr{F}_{0}$ is a generic subset of $H$, in particular $B_{i_{0}}$ contains $H$. Let $V$ be a Sylow $U_{K}$-subgroup of $B_{i_{0}}$ containing $H$. For each $F \in \mathscr{F}_{0}$, let $V_{F}$ be the intersection of the Sylow $U_{K}$-subgroups of $B_{i_{0}}$ containing $F$. Then, for each $F \in \mathscr{F}_{0}, V_{F}$ is normalized by $N_{G}(F)^{\circ}$ and we obtain $N_{V_{F}}(F) \leq F\left(N_{G}(F)^{\circ}\right) \leq F\left(N_{G}(F)\right)$. As each $U_{K}$-subgroup of $B_{i_{0}}$ is homogeneous, this implies $N_{V_{F}}(F) \leq V_{K}(F)=F$, and the nilpotence of $V_{F}$ gives $V_{F}=F<H$. In particular, for each $F \in \mathscr{F}_{0}$, there exists a Sylow $U_{K}$-subgroup $S_{F}$ of $B_{i_{0}}$ such that $F \leq S_{F}$ and $S_{F} \cap H<H$. By Fact 6.5 , for each $F \in \mathscr{F}_{0}$, there exists $x_{F} \in B_{i_{0}}$ such that $S_{F}=V^{x_{F}}$ and Proposition 10.3 shows that the family $\left\{H \cap S_{F} \mid F \in \mathscr{F}_{0}\right\}$ is finite. Since, for each $F \in \mathscr{F}_{0}$, we have $F \leq H \cap S_{F}<H$, this contradicts the genericity of $\cup \mathscr{F}_{0}$ in $H$. Hence the set $\mathscr{B}=\left\{B_{i} \mid i \in I\right\}$ is infinite.

Suppose that $C$ is abelian. By Lemma $10.4, N_{G}(H)^{\circ}$ centralizes $H$ and, for each $i \in I$, we have $C \leq N_{G}(H)^{\circ}=C_{G}(H)^{\circ} \leq N_{G}\left(F_{i}\right)^{\circ} \leq B_{i}$. Since $\mathscr{B}$ is infinite, Fact 6.9 gives $C=N_{G}(H)^{\circ}$ and $H=V_{K}(H)=U_{K}\left(F\left(N_{G}(H)\right)\right)=U_{K}(C)$, contradicting $H<U_{K}(C)$. Hence $C$ is nonabelian and $C=U_{K}(C)$ (Fact 6.8). Moreover, by Corollary 8.3, there is a unique maximal $C$-subgroup $B$ of $G$ containing $C$, and $B$ is a solvable $\widetilde{V}$-subgroup. In particular its nilpotent definable subgroups are $\widetilde{U}$-subgroups (Corollary 3.17).

We suppose $N_{G}(H)^{\circ} \leq B$. Then Fact 2.14 says that $E_{B}(H)$ is a connected definable subgroup such that $H \leq F\left(E_{B}(H)\right)$, so $H \leq U_{K}\left(F\left(E_{B}(H)\right)\right)$. But $U_{K}\left(F\left(E_{B}(H)\right)\right)$ is a homogeneous $U_{K}$-subgroup (Fact 3.13) and $E_{B}(H)$ contains $C$ and $N_{G}(H)^{\circ}$, so $N_{U_{K}\left(F\left(E_{B}(H)\right)\right)}(H)$ is contained in $U_{K}\left(F\left(N_{G}(H)^{\circ}\right)\right)=V_{K}(H)=$ $H$. Now Fact 6.3 shows that $H$ is a Sylow $U_{K}$-subgroup of $U_{K}\left(F\left(E_{B}(H)\right)\right)$, consequently $H=U_{K}\left(F\left(E_{B}(H)\right)\right)$. Thus $H$ is normal in $E_{B}(H)$ and $E_{B}(H)=N_{G}(H)^{\circ}$, in particular $N_{G}(H)^{\circ}$ contains $C$. If $N_{G}(H)^{\circ} \leq N_{G}(C)$, then we have $N_{G}(H)^{\circ}=C$ and $H=V_{K}(H)=U_{K}(F(C))=U_{K}(C)=C$, contradicting $H<C$, so there exists $g \in N_{G}(H)^{\circ} \backslash N_{G}(C)$. Consequently we have $g \in B \backslash N_{B}(C)$ and $H \leq C^{g}$, so we may assume $N_{G}(H)^{\circ} \nless \leq B$.

Since $H$ is nilpotent, $N_{G}(H)^{\circ}$ is a $C$-subgroup, and Corollary 8.3 shows that $N_{C}(H)$ is abelian, so $U_{K}\left(\left(N_{G}(H)^{\circ}\right)^{\prime}\right) \leq U_{K}\left(X_{K}(H)\right)$ is trivial (Lemmas 9.3 and 10.4). Moreover $N_{G}(H)^{\circ}$ centralizes $H$ (Lemma 10.4) and, for each $i \in I$, we obtain $N_{G}(H)^{\circ}=C_{G}(H)^{\circ} \leq N_{G}\left(F_{i}\right)^{\circ} \leq B_{i}$. If $N_{G}(H)^{\circ}$ is abelian then, since $N_{G}\left(N_{G}(H)^{\circ}\right)^{\circ} \leq N_{G}\left(V_{K}(H)\right)^{\circ}=N_{G}(H)^{\circ}$, it is an Carter subgroup of $G$, and Theorem 8.5 says that it is conjugate to $C$. This contradicts that $C$ is not abelian, so $N_{G}(H)^{\circ}$ is not abelian. Consequently, since each $C$-subgroup of $G$ containing $H$ is solvable (Proposition 8.2), Fact 6.1 provides an interpretable field $L$ of characteristic zero such that $\left(N_{G}(H)^{\circ}\right)^{\prime}$ is a nontrivial homogeneous $U_{L}$-subgroup and $U_{L}\left(C_{G}\left(N_{G}(H)^{\circ}\right)\right) \neq 1$. In particular, since $U_{K}\left(\left(N_{G}(H)^{\circ}\right)^{\prime}\right)=1$, we have $K \neq L$. 
Let $U_{L}=U_{L}\left(C_{G}\left(N_{G}(H)^{\circ}\right)\right)$. If $N_{C}\left(N_{C}(H)\right)$ is abelian, then $N_{C}\left(N_{C}(H)\right)$ is contained in $N_{C}(H)$, and $N_{C}(H)=C$ since $C$ is nilpotent, contradicting that $C$ is nonabelian, so $N_{C}\left(N_{C}(H)\right)$ is nonabelian. Since $N_{C}(H)$ is abelian, $N_{G}\left(N_{C}(H)\right)^{\circ}$ is a $C$-subgroup containing $N_{C}\left(N_{C}(H)\right)$, and Corollary 8.3 gives $N_{G}\left(N_{C}(H)\right)^{\circ} \leq B$. But we have $C_{G}\left(N_{G}(H)^{\circ}\right) \leq N_{G}\left(N_{C}(H)\right)$, so $B$ contains the $U_{L}$-subgroup $U_{L}$ and, since $C$ is a homogeneous $U_{K}$-subgroup such that $C=N_{G}(C)^{\circ}$, there exists $g \in U_{L} \backslash N_{G}(C) \subseteq B \backslash N_{B}(C)$. But $g \in U_{L}$ centralizes $N_{C}(H) \leq N_{G}(H)^{\circ}$, hence we have $H \leq N_{C}(H)=N_{C}(H)^{g} \leq C^{g}$, and this finishes the proof.

Theorem 10.6. - There is a uniformly definable family $\mathscr{F}=\left\{F_{i} \mid i \in I\right\}$ of proper subgroups of $U_{K}(C)$ such that $I$ is an interpretable set and:

- $F=V_{K}(F)$ for each $F \in \mathscr{F}$;

- there exist three integer $f, u$ and $v$ such that, for each $F \in \mathscr{F}, r k(F)=f$, $r k\left(N_{U_{K}(C)}(F)\right)=u$ and $r k\left(N_{G}(F)\right)=v, r k\left(F X_{K}(F)\right)=f-u+v$ and $r k(I)=r k\left(U_{K}(C)\right)-f ;$

- for any generic subset $J$ of $I, \cup_{j \in J} F_{j}$ is generic in $U_{K}(C)$;

- for each $F \in \mathscr{F}$, the following set is non generic in $F X_{K}(F)$ :

$$
F X_{K}(F) \cap\left(\bigcup_{E \in \mathscr{F},} \bigcup_{g \in G, E^{g} \neq F}\left(E X_{K}(E)\right)^{g}\right)
$$

- for any generic subset $J$ of $I, \bigcup_{j \in J,}\left(F_{j} X_{K}\left(F_{j}\right)\right)^{g}$ is generic in $G$;

- for each definable generic subset $G^{*}$ of $G$, there exist $x \in G^{*}$ and $g \in G$ such that $U_{K}(d(x))$ is nontrivial and contained in $U_{K}\left(C^{g}\right)$.

ProOF - Let $U=U_{K}(C)$,

$V=\left\langle U_{L}(C)\right| L \neq K$ is an interpretable field of characteristic 0$\rangle$

and $\mathscr{F}_{U}=\left\{U \cap U^{g} \mid 1<U \cap U^{g}<U\right\}$. For each $g \in G \backslash N_{G}(U)$, Fact 3.13 gives

$$
C \cap C^{g}=\left(U \cap U^{g}\right) \times\left(V \cap V^{g}\right) \subseteq\left(\left(\cup \mathscr{F}_{U}\right) \cup\{1\}\right) \times V
$$

Also, for each $g \in N_{G}(U) \backslash N_{G}(C), C^{g}$ is contained in the solvable subgroup $N_{G}(U)^{\circ}$

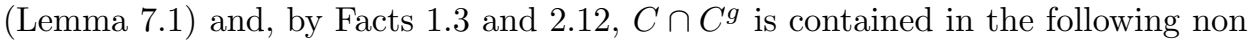
generic subset $J_{C}=C \cap\left(\cup_{g \in N_{G}(U)^{\circ} \backslash N_{G}(C)} C^{g}\right)$. Thus, if $\cup \mathscr{F}_{U}$ is non generic in $U$, then we have $C \cap\left(\cup_{g \notin N_{G}(C)} C^{g}\right) \subseteq J_{C} \cup\left(\left(\left(\cup \mathscr{F}_{U}\right) \cup\{1\}\right) \times V\right)$ and $C$ is generically disjoint of its conjugates, contradicting its non generosity. This proves that $\cup \mathscr{F}_{U}$ is a generic subset of $U$, in particular we have $\mathscr{F}_{U} \neq \emptyset$ and $1 \in \cup \mathscr{F}_{U}$.

If we let $X_{i}=\left\{g \in G \mid r k\left(U \cap U^{g}\right)=i\right\}$ for each integer $i$, then $X_{i}$ is definable for each $i$ and we have $\mathscr{F}_{U}=\cup_{i=1}^{r k(U)-1}\left\{U \cap U^{g} \mid g \in X_{i}\right\}$. Therefore there is an integer $f \in\{1, \cdots, r k(U)-1\}$ such that $\cup\left\{U \cap U^{g} \mid g \in X_{f}\right\}$ is a generic subset of $U$. In the same way, we find some integers $u$ and $v$ such that, if $Y=\{g \in$ $\left.X_{f} \mid r k\left(N_{U}\left(U \cap U^{g}\right)\right)=u, r k\left(N_{G}\left(U \cap U^{g}\right)\right)=v\right\}$, then $\cup\left\{U \cap U^{g} \mid g \in Y\right\}$ is generic in $U$.

- If $C$ is abelian, we consider the definable subset $X=Y$ of $G$ and the uniformly definable family $\mathscr{F}=\left\{U \cap U^{g} \mid g \in X\right\}$ of subgroups of $U$. In particular, for each $x \in N_{G}(U)$ and each $F \in \mathscr{F}$, we have $X^{x}=X$ and $F^{x} \in \mathscr{F}$.

- If $C$ is not abelian, then $C$ is contained in a unique maximal $C$-subgroup $B$ of $G$ (Corollary 8.3), moreover $B$ is a solvable $\widetilde{V}$-group such that $\bar{r}_{\min }(B)=$ $r$ (Proposition 8.2) and $C=U$ is a homogeneous $U_{K}$-subgroup (Fact 6.8). 
Hence, by Proposition 10.3, $C \cap\left(\cup_{b \in B \backslash N_{B}(C)} C^{b}\right)$ is a definable non generic subset of $C$. Thus, if we consider $X=\left\{y \in Y \mid U \cap U^{y} \nsubseteq \cup_{b \in B \backslash N_{B}(C)} C^{b}\right\}$ and the uniformly definable family $\mathscr{F}=\left\{U \cap U^{g} \mid g \in X\right\}$ of subgroups of $U$, the subset $\cup \mathscr{F}$ is generic in $U$. Moreover, for each $x \in N_{G}(U)$ and each $F \in \mathscr{F}$, we have $x \in N_{G}(B)$ by the unicity of $B$, so $\left(\cup_{b \in B \backslash N_{B}(C)} C^{b}\right)^{x}=$ $\cup_{b \in B \backslash N_{B}(C)} C^{b}$ and, consequently, $X^{x}=X$ and $F^{x} \in \mathscr{F}$.

Now, $\mathscr{F}$ is a uniformly definable family of nontrivial proper subgroups of $U$ such that $\cup \mathscr{F}$ is generic in $U$ and the set $\mathscr{F}=\left\{U \cap U^{g} \mid g \in X\right\}$ can be indexed by an interpretable set $I$ such that each $i \in I$ defines just one element of $\mathscr{F}=\left\{F_{i} \mid i \in\right.$ $I$ \}. Moreover, since $U$ is a homogeneous Sylow $U_{K}$-subgroup of $G$ (Fact 3.13 and Corollary 3.17), we have $U=V_{K}(U)$, so $F=V_{K}(F)$ for each $F \in \mathscr{F}$ (Corollary 9.6). Also Lemma 10.4 shows that, for each $F \in \mathscr{F}, r k\left(F X_{K}(F)\right)=f-u+v$ and $r k\left(X_{K}(F)\right)=v-u$.

By Lemma 8.11 . and 2., for each $F \in \mathscr{F}, N_{G}(F)^{\circ}$ is a solvable $\widetilde{V}$-group and $\bar{r}_{\text {min }}\left(N_{G}(F)\right)=r$, so Lemma 9.3 gives $F X_{K}(F)=F \times X_{K}(F)$ and $U_{K}\left(X_{K}(F)\right)=$ 1, in particular $F$ is the unique Sylow $U_{K}$-subgroup of $F X_{K}(F)$.

(1) For each $F \in \mathscr{F}$, the following set is non generic in $F$ :

$$
A_{F}=F \cap\left(\bigcup_{E \in \mathscr{F}, g \in G, E^{g} \neq F} E^{g}\right) .
$$

We suppose toward a contradiction that $F \in \mathscr{F}$ is a counterexample to (1). Since Corollary 9.6 gives $F \cap E^{g}=V_{K}\left(F \cap E^{g}\right)$ for each $E \in \mathscr{F}$ and each $g \in G$, Theorem 10.5 says that $C$ is a nonabelian $U_{K^{-}}$-subgroup, and there are a solvable connected definable subgroup $B_{0}$ containing $C$ and $b \in B_{0} \backslash N_{B_{0}}(C)$ such that $F \leq C^{b}$. By the unicity of $B$, we have $B_{0} \leq B$ and $b \in B$, and the choice of $\mathscr{F}$ in the nonabelian case yields a contradiction. in $U$.

(2) $r k(I)=r k(U)-f$ and, for each generic subset $J$ of $I, \cup_{j \in J} F_{j}$ is generic

We consider $U_{F}=F \backslash A_{F}$ for each $F \in \mathscr{F}$. For each $F \in \mathscr{F}$, (1) provides $r k\left(U_{F}\right)=f$. Since, for each $(E, F) \in \mathscr{F} \times \mathscr{F}$, we have $U_{E} \cap U_{F}=\emptyset$ if $E \neq F$, we obtain $r k\left(\cup_{j \in J} U_{F_{j}}\right)=r k(J)+f=r k(I)+f$ and $r k\left(\cup_{i \in I \backslash J} U_{F_{i}}\right)=r k(I \backslash J)+$ $f$. On the other hand, (1) gives $r k\left(A_{F}\right)<f$ for each $F \in \mathscr{F}$, and we obtain $r k\left(\cup_{F \in \mathscr{F}} A_{F}\right) \leq r k(I)+(f-1)$, so we find

$$
\begin{aligned}
r k(U) & =\max \left\{r k\left(\cup_{j \in J} U_{F_{j}}\right), r k\left(\cup_{i \in I \backslash J} U_{F_{i}}\right), r k\left(\cup_{F \in \mathscr{F}} A_{F}\right)\right\} \\
& =r k\left(\cup_{j \in J} U_{F_{j}}\right) \\
& =r k(I)+f .
\end{aligned}
$$

Since $r k\left(\cup_{j \in J} U_{F_{j}}\right) \leq r k\left(\cup_{j \in J} F_{j}\right) \leq r k(U)$, the preceding equality provides (2).

(3) For each $F \in \mathscr{F}$, the following set is non generic in $F X_{F}(F)$ :

$$
F X_{K}(F) \cap\left(\bigcup _ { E \in \mathscr { F } , } \left(E \in G, E_{K}^{g} \neq F\right.\right.
$$

Suppose toward a contradiction that $F \in \mathscr{F}$ is a counterexample to (3). Since, for each $E \in \mathscr{F}, E$ is the unique Sylow $U_{K}$-subgroup of $E X_{K}(E)$, for each $g \in G$ and each $E \in \mathscr{F}$, we have $F X_{K}(F) \cap\left(E X_{K}(E)\right)^{g}=\left(F \cap E^{g}\right) \times\left(X_{K}(F) \cap X_{K}(E)^{g}\right)$, in particular $A_{F}$ is generic in $F$, contradicting (1). 
(4) For any generic subset $J$ of $I, \bigcup_{j \in J, g \in G}\left(F_{j} X_{K}\left(F_{j}\right)\right)^{g}$ is generic in $G$, and there is a generic subset $J_{0}$ of $J$ such that $\bigcup_{j \in J_{0}, g \in G} X_{K}\left(F_{j}\right)^{g}$ is not generic in $G$.

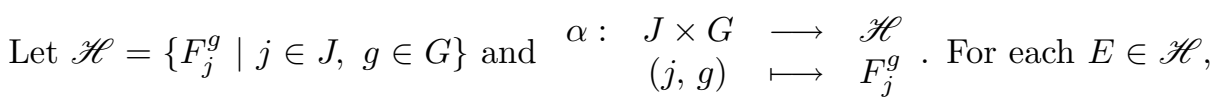
there exists $g \in G$ and $j \in J$ such that $F_{j}^{g}=E$ and we have $r k\left\{h \in G \mid F_{j}^{h}=\right.$ $E\}=r k\left(N_{G}\left(F_{j}\right)\right)=v$. Moreover there exists an integer $w$ such that $\mathscr{H}_{0}=\{E \in$ $\left.\mathscr{H} \mid r k\left(\alpha^{-1}(E)\right)=w\right\}$ is a definable generic subset of $\mathscr{H}$. Thus, if for a fixed $j_{0} \in J$ there exists $h \in G$ such that $F_{j_{0}}^{h} \in \mathscr{H}_{0}$, then for each $g \in G$ we have

$$
r k\left(\alpha^{-1}\left(F_{j_{0}}^{g}\right)\right)=r k\left(\left\{j \in J \mid \exists k \in G, F_{j}^{k}=F_{j_{0}}\right\}\right)+v .
$$

Since this equality holds for every $g \in G$, we obtain $r k\left(\alpha^{-1}\left(F_{j_{0}}^{g}\right)\right)=r k\left(\alpha^{-1}\left(F_{j_{0}}^{h}\right)\right)=$ $w$, and $F_{j_{0}}^{g} \in \mathscr{H}_{0}$ for every $g \in G$. In other words, there exists a generic subset $J_{0}$ of $J$ such that, for every $\left.\left(j_{0}, g\right) \in J_{0} \times G, F_{j_{0}}^{g}\right)$ is an element of $\mathscr{H}_{0}$, and, without loss of generality, we may assume $J=J_{0}$ and $\mathscr{H}=\mathscr{H}_{0}$.

Consequently we have $r k(J)+r k(G)-w=r k(\mathscr{H})$ and, by $(2), r k(\mathscr{H})=$ $r k(U)-f+r k(G)-w$. On the other hand, by Proposition 10.2 , for each $E \in \mathscr{H}$ there is $\left(j_{0}, g\right) \in J \times G$ such that $E=F_{j_{0}}^{g}$ and we have

$$
\begin{aligned}
r k\left\{j \in J \mid \exists h \in G, F_{j}^{h}=E\right\} & =r k\left\{j \in J \mid \exists h \in N_{G}(C), F_{j}^{h}=F_{j_{0}}\right\} \\
& \leq r k\left(N_{G}(C)\right)-r k\left(N_{N_{G}(C)}\left(F_{j_{0}}\right)\right) \\
& =r k(C)-r k\left(N_{C}\left(F_{j_{0}}\right)\right) .
\end{aligned}
$$

So we obtain $w \leq\left(r k(C)-r k\left(N_{C}\left(F_{j_{0}}\right)\right)\right)+v$. Also, by Fact 3.13, we find $r k(C)-$ $r k\left(N_{C}\left(F_{j_{0}}\right)\right)=r k(U)-r k\left(N_{U}\left(F_{j_{0}}\right)\right)=r k(U)-u$, therefore $w \leq v+r k(U)-u$. Henceforth, by $(3)$ and since $r k\left(F X_{K}(F)\right)=f-u+v$ and $r k\left(X_{K}(F)\right)=v-u$ for each $F \in \mathscr{F}$, we obtain

$$
\begin{aligned}
r k\left(\bigcup_{j \in J, g \in G}\left(F_{j} X_{K}\left(F_{j}\right)\right)^{g}\right) & \geq r k(\mathscr{H})+(f-u+v) \\
& =r k(\mathscr{H})-r k(U)+f+(v+r k(U)-u) \\
& \geq r k(\mathscr{H})-r k(U)+f+w \\
& =r k(G) .
\end{aligned}
$$

This proves the genericity of (4), and it shows that $w=v+r k(U)-u$, so

$$
\begin{aligned}
r k\left(\bigcup_{j \in J, g \in G} X_{K}\left(F_{j}\right)^{g}\right) & \leq r k(\mathscr{H})+(v-u) \\
& =(r k(U)-f+r k(G)-w)+(v-u) \\
& =(r k(U)-f+r k(G)-(v+r k(U)-u))+(v-u) \\
& =r k(G)-f .
\end{aligned}
$$

(5) For each definable generic subset $G^{*}$ of $G$, there exist $x \in G^{*}$ and $g \in G$ such that $U_{K}(d(x))$ is nontrivial and contained in $U_{K}\left(C^{g}\right)$.

We consider $J_{0}$ as in (4). Then the subset $W_{0}=\bigcup_{j \in J_{0}, g \in G} X_{K}\left(F_{j}\right)^{g}$ is not generic in $G$, and $W_{1}=\cup_{j \in J_{0}, g \in G}\left(F_{j} X_{K}\left(F_{j}\right)\right)^{g}$ is generic in $G$, so there exists $x \in G^{*} \cap\left(W_{1} \backslash W_{0}\right)$. Then $U_{L}(d(x))$ is contained in $W_{0}$ for each interpretable field $L \neq K$ of characteristic zero. Since there exist $F \in \mathscr{F}$ and $g \in G$ such that 
$x \in\left(F X_{K}(F)\right)^{g}$, Fact 3.13 shows that $U_{K}(d(x))$ is nontrivial. But $F^{g}$ is the unique Sylow $U_{K}$-subgroup of $\left(F X_{K}(F)\right)^{g}$, hence we have $U_{K}(d(x)) \leq F^{g} \leq U_{K}\left(C^{g}\right)$.

\section{CONCLUSION}

In this section, we show that Theorem 1.5 holds for any $C^{*}$-group (Theorems 11.1 and 11.3). Consequently, by Theorem 4.14, we obtain Theorem 1.5.

Theorem 11.1. $-C$ is a homogeneous $U_{K}$-subgroup.

ProOF - We assume toward a contradiction that $C$ is not a homogeneous $U_{K^{-}}$ subgroup. By Fact $6.8, C$ is abelian. We consider a uniformly definable family $\mathscr{F}$ of subgroups of $U_{K}(C)$ as in Theorem 10.6, and the subgroup

$$
\left.E=\left\langle U_{L}(C)\right| L \neq K \text { is an interpretable field of characteristic } 0\right\rangle .
$$

By Fact 3.13, we have $C=U_{K}(C) \times E$, in particular $E$ is nontrivial and $N_{G}(E)^{\circ}$ is a $C$-subgroup. Moreover, by Lemma 8.1 1. and $3 ., N_{G}(E)^{\circ}$ is a solvable $\widetilde{V}$-subgroup and $U_{K}(C)$ is a Sylow $U_{K}$-subgroup of $N_{G}(E)^{\circ}$. Let $J=\left\{j \in I \mid \forall g \in N_{G}(E)^{\circ} \backslash\right.$ $\left.N_{G}\left(U_{K}(C)\right), F_{j} \not \leq U_{K}(C)^{g}\right\}$. Since $\cup_{i \in I} F_{i}$ is generic in $U_{K}(C)$, Proposition 10.3 shows that $\cup_{j \in J} F_{j}$ is generic in $U_{K}(C)$ too, so we have $r k\left(U_{K}(C)\right) \leq r k(J)+f \leq$ $r k(I)+f=r k\left(U_{K}(C)\right)$. Thus $r k(J)=r k(I)$ and $J$ is generic in $I$, therefore $\bigcup_{j \in J, g \in G}\left(F_{j} X_{K}\left(F_{j}\right)\right)^{g}$ is generic in $G$ (Theorem 10.6).

We show that, for each $j \in J, F_{j} E$ is a Carter subgroup of $F_{j} X_{K}\left(F_{j}\right)$. Let $j \in J$. For each $g \in N_{N_{G}(E)^{\circ}}\left(F_{j}\right), F_{j}$ is contained in $U_{K}(C)^{g}$, consequently $N_{N_{G}(E)^{\circ}}\left(F_{j}\right)$ normalizes $U_{K}(C)$ by the choice of $J$. Thus, since $N_{G}\left(F_{j} E\right)^{\circ} \leq$ $N_{G}\left(F_{j}\right) \cap N_{G}(E)^{\circ}=N_{N_{G}(E)^{\circ}}\left(F_{j}\right)$, the subgroup $N_{F_{j} X_{K}\left(F_{j}\right)}\left(F_{j} E\right)^{\circ}$ normalizes $U_{K}(C) E=C$, and $N_{F_{j} X_{K}\left(F_{j}\right)}\left(F_{j} E\right)^{\circ}$ is contained in $C \cap F_{j} X_{K}\left(F_{j}\right)=F_{j}(C \cap$ $\left.X_{K}\left(F_{j}\right)\right)$. By Lemma 9.3 , we have $U_{K}\left(X_{K}\left(F_{j}\right)\right)=1$, so $U_{K}\left(C \cap X_{K}\left(F_{j}\right)\right)=1$ and $C \cap X_{K}\left(F_{j}\right)$ is contained in $E$ (Fact 3.13). This proves that $N_{F_{j} X_{K}\left(F_{j}\right)}\left(F_{j} E\right)^{\circ}$ is contained in $F_{j} E$ and, since $E \leq X_{K}\left(F_{j}\right), F_{j} E$ is a Carter subgroup of $F_{j} X_{K}\left(F_{j}\right)$.

We consider $X=\cup_{g \in G} C^{g}$. Then the subset $Y=\left(\bigcup_{j \in J, g \in G}\left(F_{j} X_{K}\left(F_{j}\right)\right)^{g}\right) \backslash X$ is generic $G$. On the other hand, the preceding paragraph and Fact 2.12 show that, for each $j \in J$, we have $r k\left(F_{j} X_{K}\left(F_{j}\right) \backslash X\right)<r k\left(F_{j} X_{K}\left(F_{j}\right)\right)=f-u+v$. Hence, since $N_{G}\left(F_{j} X_{K}\left(F_{j}\right)\right)=N_{G}\left(F_{j}\right)$ for each $j \in J$, we have

$$
\begin{aligned}
r k(Y) & <(f-u+v)+r k(J)+(r k(G)-v) \\
& =(f-u+v)+r k(I)+(r k(G)-v) \\
& =(f-u+v)+\left(r k\left(U_{K}(C)\right)-f\right)+(r k(G)-v) \\
& =(f-u+v)+(u-f)+(r k(G)-v) \\
& =r k(G),
\end{aligned}
$$

which contradicts the genericity of $Y$.

Lemma 11.2. - Let $H$ be a nontrivial definable subgroup of $U_{K}(C)$ and suppose $H=V_{K}(H)$. Then $N_{U_{K}(C)}(H)$ is a Sylow $U_{0, r}$-subgroup of $N_{G}(H)$.

Proof - Let $N=N_{C}(H)$. By Theorem 11.1, $C$ is a homogeneous $U_{K}$-subgroup, in particular $C=U_{K}(C)$ and $N$ is a $U_{K}$-subgroup of $N_{G}(H)$. Moreover, since $r k(K)=r, N$ is a $U_{0, r}$-subgroup and there is a Sylow $U_{0, r}$-subgroup $S$ of $N_{G}(H)$ containing $N$. By Proposition 8.4, $U_{K}(S)$ is a homogeneous $U_{K}$-subgroup, and Lemma 9.3 yields $U_{K}(S) \cap X_{K}(H)=1$. Then Lemma 10.4 shows that $N=U_{K}(S)$. 
Let $Z=Z(C)$. Since $C$ is a nontrivial homogeneous $U_{K}$-subgroup, $Z$ is a nontrivial $U_{K}$-subgroup. Since $Z$ is contained in $N=N_{C}(H), N$ centralizes $Z$, and $S$ centralizes $Z$ too (Fact 3.10). Hence $C_{G}(Z)^{\circ}$ is a $C$-subgroup containing $S$ and $C$. By Lemma 4.9 and Fact 3.4, there exist $c \in C_{G}(Z)^{\circ}$ such that $S^{c} \leq C$, in particular $S$ is a $U_{K}$-subgroup and $S=U_{K}(S)=N$. Consequently $N$ is a Sylow $U_{0, r}$-subgroup of $N_{G}(H)$.

Theorem 11.3. - $G$ has no generous Carter subgroup, and the divisible Carter subgroups of $G$ are conjugate.

PROOF - We assume toward a contradiction that $G$ has a generous Carter subgroup $E$. We consider a uniformly definable family $\mathscr{F}$ of subgroups of $U_{K}(C)$ as in Theorem 10.6. Then there exist $x \in E$ and $g \in G$ such that $U_{K}(d(x))$ is nontrivial and contained in $U_{K}\left(C^{g}\right)$, and Theorem 8.5 provides a contradiction.

Let $D$ be a divisible Carter subgroup of $G$. We show that $C$ and $D$ are conjugate. By the preceding paragraph, $D$ is non generous, and Proposition 4.7 shows that $D$ is torsion-free, and has no nontrivial pseudo-torus. By Theorem 11.1, there is an interpretable field $L$ of characteristic zero such that $D$ is a homogeneous $U_{L^{-}}$ subgroup.

We consider a uniformly definable family $\mathscr{F}=\left\{F_{i} \mid i \in I\right\}$ of nontrivial subgroups of $U_{K}(C)$ as in Theorem 10.6. Then $\cup_{i \in I, g \in G}\left(F_{i} X_{K}\left(F_{i}\right)\right)^{g}$ is generic in $G$, and Theorem 10.6 applied to $D$ yields $(i, g) \in I \times G$ and $x \in\left(F_{i} X_{K}\left(F_{i}\right)\right)^{g}$ such that $U_{L}(d(x))$ is a nontrivial subgroup of $D$. We may assume $g=1$. Now $Y=F_{i} X_{K}\left(F_{i}\right)$ is a $C$-subgroup of $G$ such that $Y \cap U_{K}(C) \neq 1$ and $Y \cap U_{L}(D) \neq 1$, therefore Proposition 8.2 says that $Y$ is solvable, and gives $\bar{r}_{\min }(Y)=r$ and $\bar{r}_{\text {min }}(Y)=\bar{r}_{\text {min }}(D)$. This proves that $r k(L)=\bar{r}_{\text {min }}(D)=r$, in particular $U_{L}(d(x))$ is a nontrivial $U_{0, r}$-subgroup of $Y \leq N_{G}\left(F_{i}\right)$. Since $N_{G}\left(F_{i}\right)^{\circ}$ is a $C$-group, it is solvable (Proposition 8.2), and Lemma 11.2 and Fact 3.4 yield $h \in N_{G}\left(F_{i}\right)^{\circ}$ such that $U_{L}(d(x))^{h} \leq N_{C}\left(F_{i}\right) \leq C$, hence we have $D^{h} \cap C \neq 1$. Now Theorem 8.5 shows that $C$ and $D$ are conjugate.

\section{REFERENCES}

[1] A.V. Borovik, J. Burdges And G. Cherlin. Involutions in groups of finite Morley rank of degenerate type. Preprint (septembre 2005).

[2] A.V. Borovik, J. Burdges And G. Cherlin. Simple groups of finite Morley rank of unipotent type, in Algebra, Logic, Set Theory, Festschrift fuer Ulrich Felgner zum 65. Studies in Logic, Vol. 4 (2007), 47-62.

[3] A.V. Borovik And A. Nesin. Groups of Finite Morley Rank. Oxford University Press, 1994.

[4] J. Burdges. A signalizer functor theorem for groups of finite Morley rank. J. Algebra 274 (2003), 215-229.

[5] J. Burdges. Sylow theory for $p=0$ in solvable groups of finite Morley rank. J. Group Theory 9 (4), 2006, 467-481.

[6] J. Burdges. The Bender method in groups of finite Morley rank. J. Algebra 312 (2007), $33-55$.

[7] G. Cherlin. Good tori in groups of finite Morley rank. J. Group Theory 8 (5), 2005, 613-622.

[8] G. Cherlin And E. Jaligot. Tame minimal simple groups of finite Morley rank. J. Algebra 276 (2004), 13-79

[9] O. Frécon. Around unipotence in groups of finite Morley rank. J. Group Theory 9 (3), 2006, 341-359.

[10] O. FréCon. Carter subgroups in tame groups of finite Morley rank. J. Group Theory $\mathbf{9}$ (3), 2006, 361-367.

[11] O. FréCon. Groupes géométriques de rang de Morley fini. Submitted. 
[12] O. FrÉCon. Pseudo-tori and subtame groups of finite Morley rank. Submitted.

[13] O. FrÉCON. Sous-groupes anormaux dans les groupes de rang de Morley fini résolubles. J. Algebra 229 (2000), 118-152.

[14] O. FréCON AND E. JALIGOT. The existence of Carter subgroups in groups of finite Morley rank. J. Group Theory 8 (5), 2005, 623-644.

[15] E. Jaligot. Generix never gives up. J. Symbolic Logic 71 (2006), 599-610.

[16] A. Pillay and W. Y. Pong. On Lascar rank and Morley rank of definable groups in differentially cloed fields. J. Symbolic Logic 67 (2002), 1189-1196.

[17] B. Poizat. Groupes stables. Nur Al-mantiq Wal-Ma'rifah, 1987.

[18] F. O. Wagner. Fields of finite Morley rank. J. Symbolic Logic 66 (2001), 703-706.

[19] F. O. Wagner. Nilpotent complements and Carter subgroups in stable $\Re$-groups. Arch. Math. Logic 33 (1994), no. 1, 23-34.

[20] F. O. Wagner. Stable groups. London Mathematical Society Lecture Note Series 240. Cambridge University Press, Cambridge, 1997. 